\title{
DECAY ESTIMATES FOR ONE-DIMENSIONAL WAVE EQUATIONS WITH INVERSE POWER POTENTIALS
}

\author{
O. COSTIN AND M. HUANG
}

\begin{abstract}
We study the one-dimensional wave equation with an inverse power potential that equals const. $x^{-m}$ for large $|x|$, where $m$ is any positive integer greater than or equal to 3 . We show that the solution decays pointwise like $t^{-m}$ for large $t$, which is consistent with existing mathematical and physical literature under slightly different assumptions.

Our results can be generalized to potentials consisting of a finite sum of inverse powers, the largest of which being const. $x^{-\alpha}$, where $\alpha>2$ is a real number, as well as potentials of the form const. $x^{-m}+O\left(x^{-m-\delta_{1}}\right)$ with $\delta_{1}>3$.
\end{abstract}

\section{INTRODUCTION}

There is an extensive literature - both mathematical and physical - on the decay estimates for wave equations and Schrödinger equations with a potential, starting with Strichartz's work for $V=0[13,14$. The case $V \neq 0$, important in physics, is the subject of many recent papers where a variety of modern analytical tools and different assumptions on $V$ are used (see [1,7-10] and also [12 for a survey).

In the physical community the corresponding problem goes by the name of tails, and the precise description of these tails is an important issue in scattering theory. Based on nonrigorous and numerical methods, physicists predicted that the solutions to wave equations on the line with potentials decaying like $|x|^{-\alpha}$ as $|x| \rightarrow \infty$ will decay in time like $t^{-\alpha}$; see for example [3,4]. Mathematically, a recent study by R. Donninger and W. Schlag ([7]) showed that for potentials $V(x)$ decaying like $|x|^{-\alpha}$ where $2<\alpha \leqslant 4$ with no bound state or zero energy resonance, the solution $\psi$ to the one-dimensional wave equation

$$
\frac{\partial^{2} \psi(x, t)}{\partial t^{2}}-\frac{\partial^{2} \psi(x, t)}{\partial x^{2}}+V(x) \psi(x, t)=0
$$

is bounded by $t^{-\alpha}$ for large $t$. They also obtained a similar estimate for the more important Regge-Wheeler potential, though it is not known whether the estimate is sharp (see [10]).

The purpose of this paper is to give sharp estimates for the decay of $\psi$ where $V(x)=$ const. $x^{-m}$ for large $|x|$ (the constants are allowed to be different for positive and negative $x$ ), where $m \in \mathbb{N}$ and $m \geqslant 3$. The result is consistent with [7] and confirms the predictions by physicists.

Our method is based on the inverse Laplace transform of the equation in $t$, a technique first used to study the time decay of Schrödinger equations (see e.g. [5, 6]), and it can be applied to potentials consisting of a finite sum of inverse powers, the largest of which being const. $x^{-\alpha}$, where $\alpha>2$ is a real number, as well as potentials

Received by the editors July 19, 2013 and, in revised form, October 16, 2013.

2010 Mathematics Subject Classification. Primary 35L05, 35P25, 34M37, 34M40, 35Q75. 
of the form const. $x^{-m}+O\left(x^{-m-\delta_{1}}\right.$ ) with $\delta_{1}>3$ (see Section 7). The advantage of our approach is that it gives sharp estimates based on explicit calculations. A further refinement of this approach is expected to lead to a proof of Price's Law on Schwarzschild black holes (see e.g. [10]).

\section{Setting And Main RESUlts}

We analyze the wave equation (1) under the following assumptions:

Assumption 1. (i) The potential $V$ is such that the one-dimensional Schrödinger operator $A:=-\frac{d^{2}}{d x^{2}}+V(x)$ has no bound states and no zero energy resonances.

(ii) $V$ is $m+2$ times differentiable.

(iii) As $x \rightarrow \pm \infty$ we have $V(x)=$ const ${ }_{.} x^{-m}$, where $m \in \mathbb{N}$ and $m \geqslant 3$.

The solution to (11) (cf. 7]) can be written as

$$
\psi(t)=\cos (t \sqrt{A}) \psi_{0}+\frac{\sin (t \sqrt{A})}{\sqrt{A}}, \psi_{1} \quad \psi_{0}(x):=\psi(x, 0), \psi_{1}(x)=\frac{\partial \psi(x, 0)}{\partial t},
$$

where $\psi_{0,1} \in L^{2}(\mathbb{R})$.

Our main results are

Theorem 1. Under Assumption 1 we have

$$
\begin{gathered}
\frac{\sin (t \sqrt{A})}{\sqrt{A}} \psi_{1}(x)=\hat{r}_{1}(x)\langle t\rangle^{-m}+\langle t\rangle^{-m} R_{1}(x, t), \\
\cos (t \sqrt{A}) \psi_{0}(x)=\hat{r}_{0}(x)\langle t\rangle^{-m-1}+\langle t\rangle^{-m-1} R_{0}(x, t),
\end{gathered}
$$

where

$$
\begin{gathered}
\left\|\langle x\rangle^{-2} \hat{r}_{j}(x)\right\|_{\infty} \lesssim\left\|\langle x\rangle^{2} \psi_{j}(x)\right\|_{1}, j=0,1, \\
\left\|\langle x\rangle^{-m-2} R_{1}(x, t)\right\|_{\infty} \leqslant\left\|\langle x\rangle^{m+2} \psi_{1}(x)\right\|_{1}, \\
\left\|\langle x\rangle^{-m-3} R_{0}(x, t)\right\|_{\infty} \leqslant\left\|\langle x\rangle^{m+3} \psi_{0}(x)\right\|_{1}+\left\|\langle x\rangle^{m+3} \psi_{0}^{\prime}(x)\right\|_{1} .
\end{gathered}
$$

Here $\langle x\rangle:=\left(1+x^{2}\right)^{1 / 2}$, and for $j=1,2$ the infinity norms of $R_{j}(x, t), j=1,2$, are in both $x$ and $t$, and $\lim _{t \rightarrow \infty} R_{j}(x, t)=0$. Moreover, $\hat{r}_{j}(x)$ are nonzero for generic initial data (cf. Remark 6).

In Section 7 we discuss generalizations where $V$ is a sum of inverse powers, and an extension of results of the type in [12. The special case $m=2$ will also be briefly discussed in Note1 1 below.

The basic strategy we use is to take the Laplace transform in $t$ of (11) and study the solutions of the transformed equation. Laplace transformability is shown in Proposition 14 in the Appendix; its existence does not require Assumption 1 (i); the result of Theorem 1 is however contingent on it.

\section{Discussion of Methods AND MAIN STEPS OF PROOF}

We use integral transforms to regularize the problem. First we take the Laplace transform $(\mathcal{L})$ in $t$ of equation (1), which transforms (1) into an ODE (see (2) below) in the dual variable $\varepsilon$. The position of the singularities of the solution of (2) indicates possible exponential behavior and oscillations, while the type of singularity is related to the type of power law decay. As a very simple illustration where the duality between decay in $t$ and singularities in $\varepsilon$ is manifest, consider the 

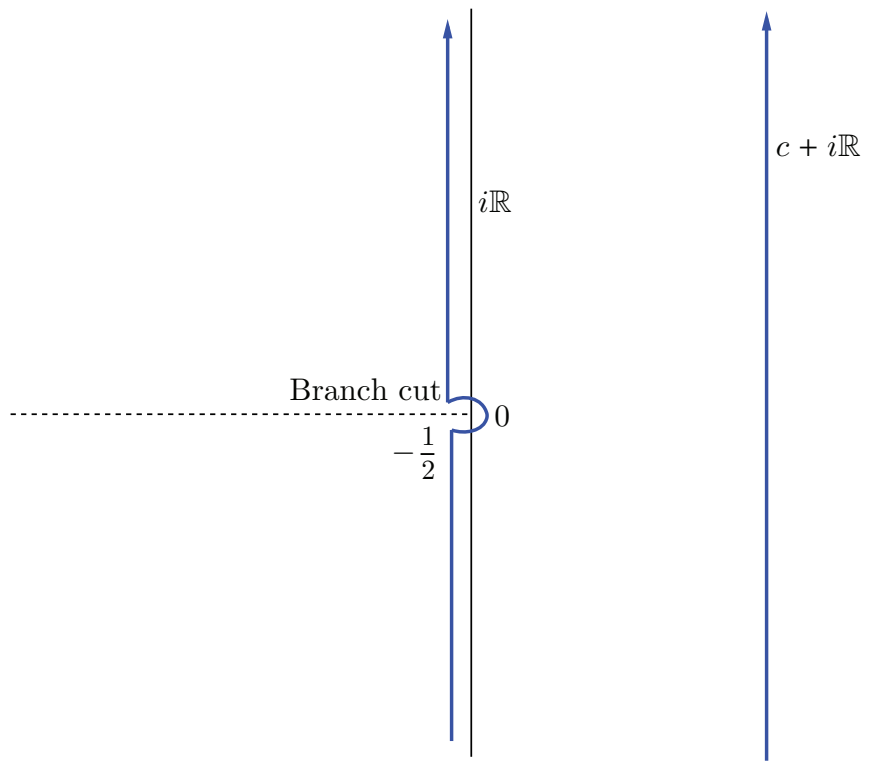

FiguRE 1. Deformation of the inverse Laplace contour for the main singular terms; the leftmost contour allows for obtaining sharp estimates using Watson's lemma. (The point $-\frac{1}{2}$ is not special-it is chosen for convenience.)

function $f(t)=t^{-\beta} e^{-\alpha t}$ with $0 \leq \beta \leq 1$ and where $\alpha$ can have a nonzero imaginary part. The Laplace transform of $f$ is

$$
\mathcal{L}(f)=\hat{f}=\Gamma(1-\beta)(\varepsilon+\alpha)^{\beta-1} .
$$

The asymptotics in $t$ follow from Watson's lemma, after deforming the contour as in Figure 1, where we took for definiteness $\varepsilon=0$. In the actual problem, the only singularity contributing to the asymptotics is indeed $\varepsilon=0$, which is a branch point. The type of the singularity follows from the small $\varepsilon$ asymptotic behavior of the associated homogenous equation (3). The asymptotic analysis in $\varepsilon$ of the full problem is complicated by the fact that when $\varepsilon$ tends to 0 and $x$ goes to $\infty$, there is a competition between $V(x)$ and $\varepsilon^{2}$, and as a result, this is effectively a singularly perturbed problem. To regularize it, we apply another transform to (3), an inverse Laplace transform in $x$. The resulting equation is the ODE (15) with only regular singular points. Global analysis of (15) involves long but conceptually relatively simple calculations, which are carried out in Section 4. The singularity structure of the Laplace transform of the solution $\psi$ of (2) is obtained in Section 5 , where the main results are the expansions (96) and (109), which are valid for all $\varepsilon \in \overline{\mathbb{H}} \backslash\{0\}$ and $x \in \mathbb{R}$. These expansions contain the crucial leading terms $r_{3}(x) \frac{\varepsilon^{m-1} \ln \varepsilon}{(1+\varepsilon\langle x\rangle)^{m+2}}$ and $r_{4}(x) \frac{\varepsilon^{m} \ln \varepsilon}{(1+\varepsilon\langle x\rangle)^{m+3}}$ that yield the desired time decay, which is shown in Section 6 . The terms in the inverse Laplace transform formula (115) are estimated differently: the contribution of the leading term is found by contour deformation (see Figure 1 ), the remainder $R(x, \varepsilon)$ is simply estimated by integration by parts, while the last term is estimated using (116). 
4. Regularity properties of the Laplace transformed EQUation

By taking the $t$-Laplace transform of (1) we obtain the ODE

$$
\hat{\psi}^{\prime \prime}(x, \varepsilon)=\left(V(x)+\varepsilon^{2}\right) \hat{\psi}(x, \varepsilon)+\psi_{1}(x)+\varepsilon \psi_{0}(x),
$$

where

$$
\hat{\psi}(x, \varepsilon)=\int_{0}^{\infty} e^{-\varepsilon t} \psi(x, t) d t .
$$

The analysis relies on properties of the exponentially decaying solutions of the homogeneous equation

$$
y^{\prime \prime}(x)=\left(V(x)+\varepsilon^{2}\right) y(x) .
$$

For $x>0$ and $\varepsilon>0$ the exponentially decaying solution has the behavior

$$
y(x)=y_{+}(x)=e^{-\varepsilon x}(1+s(x ; \varepsilon)),
$$

where $s(x ; \varepsilon)$ is an $o(1)$ power series in $1 / x$, as $x \rightarrow \infty$.

Similarly, the the exponentially decaying solution for $x<0$ and $\varepsilon>0$ satisfies

$$
y_{-}(x)=e^{\varepsilon x}\left(1+s_{-}(x ; \varepsilon)\right)=e^{-\varepsilon x}(1+o(1)) .
$$

The solution of the Laplace transformed equation (2) can be written as

$$
\begin{aligned}
\hat{\psi}(x, \varepsilon) & =\mathcal{G}\left(\psi_{1}+\varepsilon \psi_{0}\right) ; \\
\mathcal{G}(\psi) & :=\frac{1}{W(\varepsilon)}\left(y_{-}(x) \int_{\infty}^{x} y_{+}(u) \psi(u) d u-y_{+}(x) \int_{-\infty}^{x} y_{-}(u) \psi(u) d u\right),
\end{aligned}
$$

where $W=y_{+} y_{-}^{\prime}-y_{+}^{\prime} y_{-}$is the Wronskian.

Note 1. For $V(x)=a / x^{m}$ and $m=1,2$ the equation can be solved in terms of special functions.

For $V(x)=a / x^{2}$, the solution that decays like $e^{-\varepsilon x}$, as $x \rightarrow \infty$ is given in terms of the modified Bessel function $K$ as

$$
y^{+}=\sqrt{2 \varepsilon x / \pi} K_{\alpha}(\varepsilon x) ; \alpha=\sqrt{a+1 / 4} .
$$

For small $\varepsilon$ and fixed $x, y^{+}$has the form

$$
C_{1}(x) \varepsilon^{1+\alpha} A_{1}(\varepsilon)+C_{2}(x) \varepsilon^{1-\alpha} A_{2}(\varepsilon)
$$

with $A_{1}, A_{2}$ analytic.

For $m \geqslant 3$, the exponentially decaying functions have asymptotic expansions in integer powers of $\varepsilon$ and $\varepsilon \ln \varepsilon$. By symmetry it is sufficient to study the case $x>0$. The function $s$ satisfies

$$
s^{\prime \prime}-2 \varepsilon s^{\prime}-V(x) s=V(x) .
$$

It is easy to see that an $o(1)$ solution of (7) exists and is unique.

The remainder of this section is dedicated to detailed calculations to obtain the regularity properties of $y(x ; \varepsilon)$ in (4); in particular, near the branch point $\varepsilon=0$. In Section 4.1 we take the inverse Laplace transform of (7), study the transformed function $\hat{s}$, and obtain the expansions in Corollary 3. In Section 4.2 we calculate the small $\varepsilon$ behavior of $s(x ; \varepsilon)$ (expansion (42) in Lemma 5). In Section 4.3 we obtain the small $\varepsilon$ behavior of $y(x ; \varepsilon)$. Finally in Section 4.4 we obtain the regularity properties of $y$ for large $\varepsilon$. 
4.1. The inverse Laplace transform of $s(x ; \varepsilon)$ in $x$. We first assume $V$ is $m+2$ times differentiable and

$$
V(x)= \begin{cases}v_{1} x^{-m}, & x \geqslant x_{+}>1 \\ v_{2} x^{-m}, & x \leqslant x_{-}<-1\end{cases}
$$

By rescaling $x$ and $\varepsilon$ one can make $v_{1,2}= \pm 1$.

To analyze the behavior of $s(x ; \varepsilon)$ for small $\varepsilon$ and $x \geqslant x_{+}$, it is convenient to study its inverse Laplace transform, this time in $x$, to regularize the behavior at turning points. Inverse Laplace transformability does not need to be proved at this stage, since at the end we show that the Laplace transform of the solution to the dual equation solves (7). We let

$$
\hat{s}(q)=: H(q) /[q(q+2 \varepsilon)]
$$

be the formal inverse Laplace transform of $s\left(x \mapsto q\right.$ with Re $\left.x>x_{+}\right)$, and obtain

$$
H(q)=\frac{v_{1}}{(m-1) !} q^{m-1}+\mathcal{P}^{m} \frac{v_{1} H(q)}{q(q+2 \varepsilon)},
$$

where $\mathcal{P} F(q)=\int_{0}^{q} F(u) d u$. With the change of variable $q=\varepsilon \tau, H(q)=F(\tau)$, we obtain

$$
F(\tau)=\frac{v_{1} \varepsilon^{m-1} \tau^{m-1}}{(m-1) !}+\varepsilon^{m-2} \mathcal{P}^{m} \frac{v_{1} F(\tau)}{\tau(\tau+2)} .
$$

The singularity structure of $s(x ; \varepsilon)$ (cf. (7)) for small $\varepsilon$ depends on the behavior of $F(\tau)$ for large $\tau$ in the complex plane. Let

$$
\mathbb{H}:=\{z: \operatorname{Re} z>0\} ; \mathbb{H}_{1}:=\{z \in \mathbb{C}: \arg z \in(-\pi / 4,5 \pi / 4), z \neq 0\} .
$$

In the following we show that $F(\tau)$ has an asymptotic expansion in powers of $\varepsilon$, $\tau^{-1}$ and $\tau^{2-m} \ln \tau$ for small $\varepsilon$ and large $\tau$.

Lemma 1. (i) The function $\hat{s}$ has a Laplace transform in $q, F$ is analytic in $\tau$ for $\operatorname{Re} \tau>-2$ and entire in $\varepsilon$ and has the convergent expansion

$$
F(\tau)=\varepsilon^{m-1} F_{m-1}(\tau)+\sum_{j \geqslant m} \varepsilon^{j_{m}} F_{j}(\tau)
$$

where $j_{m}=(m-2)(j-m+1)+m-1, F_{m-1}(\tau)=v_{1} \tau^{m-1} /(m-1)$ !, while for $j \geqslant m$ we have

(11) $F_{j}(\tau)=\tau^{j_{m}} \sum_{n=0}^{j-m+1} \tau^{-n(m-2)}(\ln (\tau))^{n} W_{n}\left(\tau^{-1}\right) ; \quad W_{n}(z)$ analytic for $|z|<\frac{1}{2}$.

Furthermore, $F_{j}$ is analytic in $\mathbb{H}_{1}$ and

$$
\left|F_{j}\right| \lesssim \frac{|\tau|^{j_{m}}}{\left(\left(j_{m}-m+1\right) !\right)^{\frac{m}{m-2}}} \text { for } \tau \in \mathbb{H}_{1}
$$

(ii) For $|\tau| \geq 3$ we have 1

$$
\begin{aligned}
& F_{j}(\tau)=F_{j}^{[0]}(\tau)+\tau^{j_{m}-m} G_{j}(\tau), \\
& F_{j}^{[0]}(\tau):=\tau^{j m}\left(\sum_{n=0}^{m-1} a_{n, 0}^{[j]} \tau^{-n}+\sum_{k=1}^{2} \sum_{n=0}^{2-k} a_{k(m-2)+n, k}^{[j]} \tau^{-k(m-2)-n}(\ln \tau)^{k}\right),
\end{aligned}
$$

\footnotetext{
${ }^{1}$ In fact we only need to keep the first few terms of (11) and estimate the remainder.
} 
where $a_{l, k}^{[j]}=0$ if $j<m+k-1$ or $l>m$, and the following estimates hold: for some $c_{1}>0$ (independent of all indices above)

$$
\left|a_{n, l}^{[j]}\right| \leqslant \frac{c_{1}^{n}}{\left(\left(j_{m}-2 m+2\right) !\right)^{\frac{m}{m-2}}}, \quad \sup _{\tau \in \mathbb{H}_{1}}\left|(|\ln \tau|+1)^{-3} G_{j}(\tau)\right| \leqslant \frac{c_{1} j}{\left(\left(j_{m}-2 m+1\right) !\right)^{\frac{m}{m-2}}} .
$$

Proof. We analyze the case $v_{1}=1$; if $v_{1}=-1$, the arguments are very similar. We look for solutions to (9) which are $O\left(\varepsilon^{m-1} \tau^{m-1}\right)$ for small $\tau$. Consider the space $\mathcal{B}$ of functions of the form $f(\tau)=\tau^{m-1} \tilde{G}(\tau)$, where $\tilde{G}$ is analytic for, say, $|\tau|<\tau_{0}$ for arbitrarily large $\tau_{0}>0$ with the norm $\|f\|=\sup \left\{|\tilde{G}(\tau)|:|\tau|\left\langle\tau_{0} ; \tau>-a>-2\right\}\right.$. We see that this is a Banach space, and equation (91) is contractive in $\mathcal{B}$. The solution of (9) is unique, and it is analytic for small $\tau$. As a differential equation (91) reads

$$
F^{(m)}=\frac{\varepsilon^{m-2} F}{\tau(\tau+2)}
$$

The argument above, or Frobenius theory, shows that (15) also has a unique solution which is of the form $\frac{1}{(m-1) !} \varepsilon^{m-1} \tau^{m-1}(1+o(1))$ for small $\tau$. The solution is obviously analytic for $\operatorname{Re} \tau>-2$, since the only singularities of equation (9) are $\tau=0$ and $\tau=-2$, and it is entire in $\varepsilon$ for $\operatorname{Re} \tau>-2$, since the equation depends analytically on $\varepsilon$.

By standard ODE asymptotic results [15] we see that any solution of (15) is uniformly bounded in $\mathbb{C}$ by

$$
C(\varepsilon)|\tau|^{\frac{m-1}{m}} e^{\frac{m}{m-2}|\varepsilon|^{1 / m}|\tau|^{1-2 / m}}
$$

for some $C(\varepsilon)>0$. This ensures the necessary (sub)exponential bounds for taking the Laplace transform in $\varepsilon$.

We now look for solutions of (15) in the form

$$
F=\frac{\varepsilon^{m-1} \tau^{m-1}}{(m-1) !}+\sum_{j \geqslant m} \varepsilon^{j_{m}} F_{j}
$$

and we show that the expansion (17) is convergent.

The functions $F_{j}$ satisfy the recurrence

$$
F_{j+1}=\mathcal{P}^{m} \frac{F_{j}}{\tau(\tau+2)}, j \geqslant m-1 ; \quad F_{m-1}(\tau)=\tau^{m-1} /(m-1) ! .
$$

For now, we take $\tau$ in $\mathbb{H}_{1}$. It can be checked by induction that the $F_{j}$ 's are analytic in $\mathbb{H}_{1}$ and at zero, and since $\left|\tau^{j_{m}}\right| \leqslant\left|\tau^{j_{m}-1}(\tau+2)\right|$ in $\mathbb{H}_{1}$ and

$$
\left|\mathcal{P}^{m} \tau^{j_{m}-2}\right|=\frac{|\tau|^{j_{m}+m-2}}{\prod_{k=0}^{m-1}\left(j_{m}-1+k\right)} \leqslant\left(\frac{\left(j_{m}-m+1\right) !}{\left((j+1)_{m}-m+1\right) !}\right)^{\frac{m}{m-2}}|\tau|^{(j+1)_{m}},
$$

(12) follows by induction. The last inequality above comes from the fact that

$$
\left(\prod_{k=0}^{m-1}\left(j_{m}-1+k\right)\right)^{m-2} \geqslant\left(j_{m}-1\right)^{m(m-2)} \geqslant\left(\prod_{k=0}^{m-3}\left(j_{m}-m+2+k\right)\right)^{m} .
$$


It follows that the series (17) converges uniformly on any compact set in $\mathbb{H}_{1}$. Moreover, we see that the function series

$$
H(q)=\frac{q^{m-1}}{(m-1) !}+\sum_{j \geqslant m} \varepsilon^{j_{m}} F_{j}(q / \varepsilon)
$$

also converges uniformly in any compact set in $\mathbb{H}_{1}$. Existence of the Laplace transform of $\frac{H(q)}{q(q+2 \varepsilon)}$ follows from the bound (16) for $F$.

We write (9) as

$$
\tau(\tau+2) F_{j+1}^{(m)}=F_{j}, j \geqslant m-2 ; \quad F_{m-2}=0 .
$$

Note that $F_{m-1}$ is explicit (see (18)). Let $L g=\tau(\tau+2) g^{(m)}(\tau)$. Equation (21) implies

$$
L^{j-m+2} F_{j}=0, \quad j \geqslant m .
$$

Note 2. The indicial polynomial of (22) at infinity is

$$
\prod_{n=0}^{j-(m-1)} \prod_{n^{\prime}=0}^{m-1}\left(\lambda-n^{\prime}-n\right), j \geqslant m
$$

with the convention that a product is one if the index set is empty, and, by Frobenius theory, (23) implies (11). Equation (23) follows from

$$
L \tau^{\lambda}=\tau^{\lambda-m+2}\left[\lambda(\lambda-1) \cdots(\lambda-m+1)+O\left(\tau^{-1}\right)\right] .
$$

(ii) The existence of an asymptotic expansion of the form (13) follows from (11). It remains to estimate the coefficients and the remainder (which we do recursively), for which we can assume $j_{m} \geqslant 2 m-2$, since for $j_{m}<2 m-2$ the result follows directly from (11).

We have

$$
L\left(t^{n} \ln ^{l}(t)\right)=t^{n-1} \ln ^{l} t\left(1+2 t^{-1}\right)\left(n(n-1) \cdots(n-m+1)+\sum_{l=1}^{m} P_{l}(\ln t)^{-l}\right)
$$

where $P_{l}$ are polynomials of degree at most $m-1$ in $n$ and $m$ in $l$. Substituting (10) in (21) using the notation in (13) and taking $a_{n, l}^{[j]}=\left(\left(j_{m}-2 m+2\right) !\right)^{-\frac{m}{m-2}} A_{n, l}^{[j]}$, we get the following recurrence for $0 \leqslant l \leqslant 3$ with $(m-2) l \leqslant n \leqslant m-1$ :

$$
A_{n, l}^{[j]}-C_{n, l}^{[j]} A_{n, l}^{[j-1]}+\sum_{J_{n, l}} C_{n^{\prime}, l^{\prime} ; n, l}^{[j]} A_{n^{\prime}, l^{\prime}}^{[j]}=0,
$$

where $J_{n, l}$ consists of indices earlier than $n, l: J_{n, l}=\left\{\left(n, l^{\prime}\right): l^{\prime}>l\right\} \bigcup\left\{\left(n-1, l^{\prime}\right)\right.$ : $\left.0 \leqslant l^{\prime} \leqslant 2\right\}$. In (26) we have $0<C_{n, l}^{[j]}<1$, and for some $c_{4}>0$ and all $n, n^{\prime}, l, l^{\prime}, j$ we have $\left|C_{n^{\prime}, l^{\prime} ; n, l}^{[j]}\right|<c_{4}$. Solving for $A_{n, l}^{[j]}$ in the order $n=0,1, \ldots, m-1$ and for a fixed $n$ in the order $l=2,1,0$, the first inequality in (14) follows inductively on $j$.

Note 3 . Let $R_{j}=\tau^{j_{m}-m-1} G_{j}$. Then, $R_{j}$ satisfies the recurrence

$$
\tau(\tau+2) R_{j}^{(m)}=R_{j-1}+\tau^{j_{m}-2 m+1} p_{1}(\ln \tau),
$$

where $p_{1}$ is a quadratic polynomial with coefficients bounded by

$$
\frac{c_{2}}{\left[\left((j-1)_{m}-2 m+2\right) !\right]^{\frac{m}{m-2}}}
$$

for some $j$-independent $c_{2}$. 
Equation (27) simply follows by writing $F_{j}(\tau)=F_{j}^{[0]}+R_{j}(\tau)$, calculating the finite sum $L F_{j}^{[0]}$ explicitly using (24) and (25), and estimating the coefficients of $p_{1}$ using the first inequality in (14).

Proof of the last inequality in (14). Since $j_{m}=(j-1)_{m}+m-2$, we have by (27)

$$
\begin{aligned}
\left|R_{j}\right| & =\left|\mathcal{P}^{m} \frac{\tau^{(j-1)_{m}-m-1}\left(G_{j-1}(\tau)+p_{1}(\ln \tau)\right)}{\tau+2}\right| \\
& \leq \mathcal{P}^{m}\left|\tau^{(j-1)_{m}-m-2}\left(\tau G_{j-1}(\tau)+p_{1}(\ln \tau)\right)\right| .
\end{aligned}
$$

Also, by direct integration we have

$$
\int_{0}^{\tau}\left|t^{n} \ln ^{l} t\right| d t \leqslant c_{3}(n+1)^{-1}\left|\tau^{n+1}\right|\left(\left|\ln ^{l} \tau\right|+1\right), \quad l=0,1,2, n>0,
$$

for some $c_{3}$. The rest follows from (28) and (29) by induction on $j$, noting that (cf. also (19))

$$
\frac{1}{\prod_{k=0}^{m-1}\left((j-1)_{m}-m-1+k\right)} \leq\left(\frac{\left((j-1)_{m}-2 m+1\right) !}{\left(j_{m}-2 m+1\right) !}\right)^{\frac{m}{m-2}}
$$

Since $\hat{s}(q)=H(q) /[q(q+2 \varepsilon)]$ and $H(q)=F(q / \varepsilon)$, the expansions for $F$ in Lemma 1 allow us to obtain the corresponding expansions for $H$ and $\hat{s}$ :

Lemma 2. (i) For $|q| \geqslant 3|\varepsilon|$ 2 we have the expansion

$$
\begin{aligned}
H(q)= & q^{m-1} H_{0,0}(q)+\sum_{k=1}^{m-1} \varepsilon^{k} q^{2 m-3-k} H_{k, 0}(q) \\
& +\sum_{n=1}^{2} \sum_{k=0}^{2-n} \varepsilon^{n(m-2)+k} q^{m-1-k} H_{k, n}(q) \ln ^{n}(q / \varepsilon)+\varepsilon^{m} \tilde{R}(q / \varepsilon, q),
\end{aligned}
$$

where $H_{i, j}(q)(j \leqslant 2)$ are analytic in $q$ with sub-exponential growth for large $q$, $H_{0,2}=0$ if $m>3$, and $\left|\partial^{(k+l)} \tilde{R}(u, v) / \partial u^{k} \partial v^{l}\right| \lesssim(|\ln u|+1)^{3}|u|^{-k}$ for $\operatorname{Re} u \geqslant 0$, $q / \varepsilon \in \mathbb{H}_{1},|v|<$ const., and $0 \leqslant k+l \leqslant m+1$.

(ii) For $|q| \leqslant 3|\varepsilon|$ and $\operatorname{Re}(q / \varepsilon) \geqslant 0, H(q)$ is analytic in $q$, entire in $\varepsilon$, and $|H(q)| \lesssim|q|^{m-1}$.

Proof. (i) Recall that $q=\varepsilon \tau, H(q)=F(\tau)$, and $a_{l, k}^{[j]}=0$ if $j<m+k-1$ by Lemma 1. We thus substitute $\tau=q / \varepsilon$ in (13) and obtain (31) by collecting coefficients of powers of $\varepsilon$ and $\ln (q / \varepsilon)$. We define

$$
\begin{gathered}
q^{m-1} H_{0,0}(q)=\frac{q^{m-1}}{(m-1) !}+\sum_{j \geqslant m} q^{j_{m}} a_{0,0}^{[j]}, \\
q^{2 m-3-k} H_{k, 0}(q)=\sum_{j \geqslant m} a_{k, 0}^{[j]} q^{j_{m}-k}(1 \leqslant k \leqslant m-1), \\
q^{m-1-k} H_{k, n}(q)=\sum_{j \geqslant m+n-1} a_{n(m-2)+k, n}^{[j]} q^{j_{m}-(n(m-2)+k)}(1 \leqslant n \leqslant 2,0 \leqslant k \leqslant 2-n), \\
\tilde{R}(q / \varepsilon, q)=\sum_{j_{m} \geqslant m} q^{j_{m}-m} G_{j}(q / \varepsilon) .
\end{gathered}
$$

\footnotetext{
${ }^{2} 3$ can be replaced by any constant bigger than 2 .
} 
Convergence of the three series is ensured by (14). Note that the $H_{k, n}$ are analytic in $q$ for all choices of $k$ and $n$ above. Since $F_{j}(\tau)$ is analytic in $\mathbb{H}_{1}$ (see Lemma 1), by (14) and Cauchy's formula we have

$$
\left|G_{j}^{(k)}(\tau)\right| \lesssim \frac{j|\tau|^{-k}(|\ln \tau|+1)^{3}}{\left(\left(j_{m}-2 m+1\right) !\right)^{\frac{m}{m-2}}}
$$

for $\tau \in \mathbb{H}$. Noting that for $a>0$ we have $(j !)^{a}>$ const. ${ }^{j} \Gamma(a j+1)$, (14) implies

$$
\sum_{j=m}^{\infty} \frac{|\tau|^{j_{m}}}{\left(\left(j_{m}-2 m+2\right) !\right)^{\frac{m}{m-2}}} \leqslant \sum_{j=0}^{\infty} \frac{|\tau|^{j}}{(j !)^{\frac{m}{m-2}}} \lesssim \sum_{j=0}^{\infty} \frac{\left|c_{t} \tau\right|^{j}}{\Gamma\left(\frac{m}{m-2} j+1\right)} \lesssim e^{\delta|\tau|}, \quad \forall \delta>0 .
$$

Thus sub-exponential growth of $H_{i, j}$ follows. In fact it is elementary to show that the last sum is bounded by $e^{\text {const. } \tau^{\frac{m-2}{m}}}$. The rest of (i) follows from (10) using (12) and (13) to estimate the terms.

(ii) This follows from (10) and (12).

Corollary 3. (i) For $|q| \geqslant 3|\varepsilon|$ we have

$$
\begin{aligned}
\hat{s}(q) & =\frac{H(q)}{q(q+2 \varepsilon)} \\
& =\sum_{n=0}^{2} \varepsilon^{n(m-2)}\left(\frac{\varepsilon^{m-2} \tilde{H}_{1, n+1}(\varepsilon)}{q+2 \varepsilon}+\tilde{H}_{2, n+1}(q, \varepsilon)\right) \ln ^{n}(q / \varepsilon)+\varepsilon^{m} \frac{\tilde{R}(q / \varepsilon, q)}{q(q+2 \varepsilon)},
\end{aligned}
$$

where $\tilde{H}_{1, j}$ are entire, $\tilde{H}_{2, j}(q, \varepsilon)$ are entire in $\varepsilon$, analytic in $q$ and have subexponential growth for large $q, \tilde{H}_{2,1}(\varepsilon, \varepsilon)=O\left(\varepsilon^{m-3}\right), \tilde{H}_{1,1}(0)=\frac{(-2)^{m-2}}{\Gamma(m)}, \tilde{H}_{k, 3}=0$ if $m>3$, and $\tilde{R}$ is the same as in Lemma 2 .

(ii) For $|q| \leqslant 3|\varepsilon|$ and $\operatorname{Re}(q / \varepsilon) \geqslant 0$ we have

$$
\hat{s}(q)=\frac{H(q)}{q(q+2 \varepsilon)}=\frac{\varepsilon^{m-2} H_{1}(\varepsilon)}{q+2 \varepsilon}+\varepsilon^{m-3} H_{2}(q, \varepsilon),
$$

where $H_{i}$ are analytic in $q$ and entire in $\varepsilon$.

Proof. Note that for a function $f$ analytic in $q$ and entire in $\varepsilon$ we have

$$
\frac{f(q ; \varepsilon)}{q+2 \varepsilon}=\frac{f(-2 \varepsilon)}{q+2 \varepsilon}+\frac{f(q)-f(-2 \varepsilon)}{q+2 \varepsilon}:=\frac{\tilde{f}_{1}(\varepsilon)}{q+2 \varepsilon}+\tilde{f}_{2}(q ; \varepsilon),
$$

where $\tilde{f}_{2}$ is analytic in $q$ and entire in $\varepsilon$. Now using (31), we take

$$
q f(q ; \varepsilon)=q^{m-1} H_{0,0}(q)+\sum_{k=1}^{m-1} \varepsilon^{k} q^{2 m-3-k} H_{k, 0}(q)
$$

in (34) and define $\varepsilon^{(2-k)(m-2)} \tilde{H}_{k, 1}=\tilde{f}_{k}(k=1,2)$. For $k, n=1,2$ we take

$$
q f(q ; \varepsilon)=\sum_{k=0}^{2-n} \varepsilon^{k} q^{m-1-k} H_{k, n}(q)
$$

in (34) and define $\varepsilon^{(2-k+n)(m-2)} \tilde{H}_{k, n+1}=\tilde{f}_{k}(k, n=1,2)$. The sub-exponential growth of $\tilde{H}_{2, j}$ follows immediately from the sub-exponential growth of $H_{2, j}(q)$. 
In addition, $\tilde{H}_{1,1}(0)=H_{0,0}(0)=-\frac{1}{2} F_{m-1}(-2)=\frac{(-2)^{m-2}}{\Gamma(m)}$ by Lemma 1 and the proof of Lemma 2

Similarly, to obtain (33) we use (34) for $f(q)=H(q)$ and apply Lemma 2 (ii).

4.2. Asymptotic expansion of $s(x ; \varepsilon)$ for small $\varepsilon$. For small $\varepsilon$, the function $s(x ; \varepsilon)$ (cf. (44)) has an asymptotic expansion in powers of $\varepsilon$ and $\varepsilon^{m-2} \ln \varepsilon$. To obtain the time decay of $\psi$, only a few terms of this expansion are needed, and they are obtained in Lemma 4 below.

Lemma 4. (i) Let $\delta>0$ be arbitrarily small but fixed. We have for $x \geqslant x_{+}, \varepsilon \in \mathbb{H}$ and $|\varepsilon| \leqslant 1 / x$

$$
s(x ; \varepsilon)=h_{1}(x) \varepsilon^{m-2} \ln \varepsilon+h_{2}(x) \varepsilon^{m-1} \ln \varepsilon+h_{3}(x) \varepsilon^{2 m-4}(\ln \varepsilon)^{2}+Q(x ; \varepsilon),
$$

where the smooth functions $h_{j}$ satisfy

$$
h_{1}(x) \sim-\frac{(-2)^{m-2}}{\Gamma(m)}, h_{2}(x) \sim \frac{(-2)^{m-1} x}{\Gamma(m)}, h_{3}(x) \sim a_{0} \text { as } x \rightarrow \infty,
$$

where $a_{0}$ is a constant and for $m>3$ one can take $h_{3}(x)=0$. Furthermore,

$$
\sup _{0 \leqslant k \leqslant m-1}\left|x^{m-2-k} \frac{\partial^{k} Q(x ; \varepsilon)}{\partial \varepsilon^{k}}\right|<\infty ; \sup _{m \leqslant k \leqslant m+1}\left|\varepsilon^{k-m+\delta} x^{-2} \frac{\partial^{k} Q(x ; \varepsilon)}{\partial \varepsilon^{k}}\right|<\infty .
$$

The asymptotic formula (35) is twice differentiable in $x$, i.e. (37) holds with $Q$ replaced by $x Q^{\prime}$ or $x^{2} Q^{\prime \prime}$.

Proof. We write

$$
s(x ; \varepsilon)=\left(\int_{0}^{3 \varepsilon}+\int_{3 \varepsilon}^{1}+\int_{1}^{\infty}\right) \frac{H(q) e^{-q x}}{q(q+2 \varepsilon)} d q .
$$

The first and last terms are estimated easily: the last integral is manifestly analytic in $\varepsilon$ and decays exponentially in $x$, so (37) is obvious. By Corollary 3 the first integral is equal to

$$
\varepsilon^{m-2} \int_{0}^{3}\left(\frac{H_{1}(\varepsilon)}{\tau+2}+H_{2}(\varepsilon \tau, \varepsilon)\right) e^{-\varepsilon \tau x} d \tau .
$$

Thus it is analytic in $\varepsilon$ and satisfies the estimates in (37) by direct differentiation in $\varepsilon$, noting that $\left|\varepsilon^{m-2}\right| \lesssim x^{2-m}$.

To estimate the middle integral in (38) we use (32) to write

$$
\int_{3 \varepsilon}^{1} \frac{H(q) e^{-q x}}{q(q+2 \varepsilon)} d q=S_{1}(x, \varepsilon)+S_{2}(x, \varepsilon)+S_{3}(x, \varepsilon)
$$

where

$$
\begin{gathered}
S_{1}(x, \varepsilon)=\sum_{k=1}^{3} \varepsilon^{k(m-2)} \tilde{H}_{1, k}(\varepsilon) \int_{3 \varepsilon}^{1} \frac{(\ln (q / \varepsilon))^{k-1} e^{-q x}}{q+2 \varepsilon} d q, \\
S_{2}(x, \varepsilon)=\sum_{k=0}^{2} \varepsilon^{k(m-2)} \int_{3 \varepsilon}^{1}(\ln (q / \varepsilon))^{k} \tilde{H}_{2, k+1}(q, \varepsilon) e^{-q x} d q, \\
S_{3}(x, \varepsilon)=\varepsilon^{m} \int_{3 \varepsilon}^{1} \frac{\tilde{R}(q / \varepsilon, q) e^{-q x}}{q(q+2 \varepsilon)} d q .
\end{gathered}
$$


Now the fact that the middle integral in (38) has an expansion of the form (35) follows from the lemma below:

Lemma 5. The terms $S_{1}(x, \varepsilon)$ and $S_{2}(x, \varepsilon)$ have expansions of the form (35), and $S_{3}(x, \varepsilon)$ satisfies the estimates in (37). More precisely we have

$$
S_{k}(x ; \varepsilon)=h_{k, 1}(x) \varepsilon^{m-2} \ln \varepsilon+h_{k, 2}(x) \varepsilon^{m-1} \ln \varepsilon+h_{k, 3}(x) \varepsilon^{2 m-4}(\ln \varepsilon)^{2}+R_{k, 0}(x ; \varepsilon)
$$

for $k=1,2$, where $h_{k, j}$ have the large $x$ asymptotics

$$
\begin{gathered}
h_{1,1} \sim-\frac{(-2)^{m-2}}{\Gamma(m)}(1+o(1)) ; h_{1,2} \sim-\frac{(-2)^{m-2}(2 x+o(x))}{\Gamma(m)} ; h_{1,3} \sim a_{0}+o(1), \\
h_{2,1}=o(1) ; h_{2,2}=O(1) ; h_{2,3}=o(1),
\end{gathered}
$$

where $a_{0}$ is a constant and $R_{k, 0}$ satisfy the estimates in (37).

Proof. We first show the result for $S_{1}$ using (39). Since $\tilde{H}_{1, k}$ are analytic in $\varepsilon$, we only need to analyze the integrals

$$
\int_{3 \varepsilon}^{1} \frac{\ln ^{l}(q / \varepsilon) e^{-q x}}{q+2 \varepsilon} d q=-\int_{1}^{\infty} \frac{\ln ^{l}(q / \varepsilon) e^{-q x}}{q+2 \varepsilon} d q+\int_{3}^{\infty} \frac{\ln ^{l}(\tau) e^{-\varepsilon \tau x}}{\tau+2} d \tau,
$$

where $0 \leqslant l \leqslant 2$. The first integral on the right hand side is a polynomial in $\ln \varepsilon$ times a function analytic in $\varepsilon$ with exponential decay in $x$, and the last one is equal to

$$
\int_{3}^{\infty} \ln ^{l} \tau\left(\tau^{-1}-2 \tau^{-2}\right) e^{-\varepsilon \tau x} d \tau+\int_{3}^{\infty} \frac{4 \ln ^{l} \tau}{\tau^{2}(\tau+2)} e^{-\varepsilon \tau x} d \tau .
$$

To analyze the first term in (44) we need the following elementary result:

Lemma 6. Assume $l \geqslant 0, x \geqslant x_{+}$and $|\varepsilon x| \leqslant 1$. For $n \geqslant 0$ we have

$$
\int_{3}^{\infty} e^{-\varepsilon \tau x} \tau^{n}(\ln \tau)^{l} d \tau=\frac{1}{(\varepsilon x)^{n+1}} \sum_{q=0}^{l} c_{q}^{(n ; l)} \ln ^{q}(\varepsilon x)+R_{a, n}(x, \varepsilon),
$$

where $c_{q}^{(n ; l)}$ are constants with $c_{1}^{(0 ; 1)}=-1$, and $R_{a, n}$ satisfies

$$
\left|\frac{\partial^{k} R_{a, n}(x, \varepsilon)}{\partial \varepsilon^{k}}\right| \lesssim x^{k} \quad(k \geqslant 0) .
$$

In addition, for $n \leqslant-1$ we have

$$
\int_{3}^{\infty} e^{-\varepsilon \tau x} \tau^{n}(\ln \tau)^{l} d \tau=(\varepsilon x)^{-1-n} \sum_{q=0}^{l+1} c_{q}^{(n ; l)} \ln ^{q}(\varepsilon x)+R_{a, n}(x, \varepsilon),
$$

where $c_{1}^{(-1 ; 0)}=-1, c_{1}^{(-2 ; 0)}=1$, and $R_{a, n}$ satisfies (46).

The proof of this lemma is given in the Appendix.

Expansion of the first term in (44) follows directly from Lemma 6. The last term in (44) satisfies

$$
\begin{aligned}
\left|\frac{d^{k}}{d \varepsilon^{k}} \int_{3}^{\infty} \frac{4 \ln ^{l} \tau}{\tau^{2}(\tau+2)} e^{-\varepsilon \tau x} d \tau\right| \lesssim\left|x^{k} \int_{3}^{\infty} \tau^{-3+k} \ln ^{l} \tau e^{-\varepsilon \tau x} d \tau\right| & 0 \leqslant k \leqslant 1, \\
& \lesssim \begin{cases}x^{k}, & 0 \leqslant k \leqslant m+1, \\
x^{2}|\varepsilon|^{2-k}\left(1+\left|\ln ^{l+1}(\varepsilon x)\right|\right), & 2 \leqslant k\end{cases}
\end{aligned}
$$


where the second integral in (48) is estimated using Lemma 6. Thus

$$
\varepsilon^{(l+1)(m-2)+k} \int_{3}^{\infty} \frac{4 \ln ^{l} \tau}{\tau^{2}(\tau+2)} e^{-\varepsilon \tau x} d \tau
$$

satisfies (37) for all $k \geqslant 0$ and $l \geqslant 0$ by direct differentiation.

Thus combining (43) and (44) using Lemma 6] we see that

$$
\begin{aligned}
& \varepsilon^{(l+1)(m-2)} \int_{3 \varepsilon}^{1} \frac{\ln ^{l}(q / \varepsilon) e^{-q x}}{q+2 \varepsilon} d q=\varepsilon^{(l+1)(m-2)} \ln ^{l} \varepsilon \int_{1}^{\infty} \frac{e^{-q x}}{q+2 \varepsilon} d q \\
& +\sum_{n=-2}^{-1}(-2)^{-1-n} \varepsilon^{(l+1)(m-2)-1-n} x^{-1-n} \sum_{q=0}^{l+1} c_{q}^{(n ; l)} \ln ^{q}(\varepsilon x)+R_{0,0}(x, \varepsilon)
\end{aligned}
$$

where $R_{0,0}$ satisfies (37).

Letting $l=0,1,2$ in (49) we have

$$
\varepsilon^{m-2} \tilde{H}_{1, k}(\varepsilon) \int_{3 \varepsilon}^{1} \frac{e^{-q x}}{q+2 \varepsilon} d q=h_{a, 1}(x) \varepsilon^{m-2} \ln \varepsilon+h_{a, 2}(x) \varepsilon^{m-1} \ln \varepsilon+R_{0,1}(x, \varepsilon),
$$

where $h_{a, 1}(x)=-1+o(1)$ and $h_{a, 2}(x)=-2 x(1+o(1))$ for large $x$,

$\varepsilon^{2 m-4} \tilde{H}_{2, k}(\varepsilon) \int_{3 \varepsilon}^{1} \frac{\ln (q / \varepsilon) e^{-q x}}{q+2 \varepsilon} d q=c_{2}^{(-1 ; 1)} \varepsilon^{2 m-4} \ln ^{2} \varepsilon+h_{b, 1}(x) \varepsilon^{2 m-4} \ln \varepsilon+R_{0,2}(x, \varepsilon)$,

where $h_{b, 1}(x)=O(1)$ for large $x$, and

$$
\varepsilon^{3 m-6} \tilde{H}_{3, k}(\varepsilon) \int_{3 \varepsilon}^{1} \frac{\ln ^{2}(q / \varepsilon) e^{-q x}}{q+2 \varepsilon} d q=R_{0,3}(x, \varepsilon),
$$

where $R_{0, i}(0 \leqslant i \leqslant 3)$ satisfies (37). Thus (42) follows from (50), (51), and (52). Note that $\tilde{H}_{1,1}(0)=\frac{(-2)^{m-2}}{\Gamma(m)}$ by Corollary 3 ,

To show (42) for $S_{2}$ we write $\ln (q / \varepsilon)=\ln q-\ln \varepsilon$ in (40). By Corollary 3

$$
\int_{2 \varepsilon}^{1} \tilde{H}_{2, n}(q, \varepsilon) e^{-q x} d q
$$

is entire in $\varepsilon$ with its $k$-th derivative in $\varepsilon$ bounded by const. $x^{k-1}$. Thus the term containing $\tilde{H}_{2,1}$ satisfies (37) and the terms containing $\ln \varepsilon \tilde{H}_{2,2}$ and $\ln ^{2} \varepsilon \tilde{H}_{2,3}$ satisfy (42).

The term with $\ln q \tilde{H}_{2,2}$ is analyzed using integration by parts:

$$
\begin{aligned}
\varepsilon^{m-2} \int_{3 \varepsilon}^{1} \ln q \tilde{H}_{2,2}(q, \varepsilon) e^{-q x} d q= & \left.\varepsilon^{m-2} \tilde{H}_{2,2}(q, \varepsilon) e^{-q x}(q \ln q-q)\right|_{3 \varepsilon} ^{1} \\
& -\varepsilon^{m-2} \int_{3 \varepsilon}^{1}(q \ln q-q) \frac{\partial\left(\tilde{H}_{2,2}(q, \varepsilon) e^{-q x}\right)}{\partial q} d q,
\end{aligned}
$$

where the last term satisfies (37) by direct calculation and counting powers of $\varepsilon$.

The term containing $\ln q \tilde{H}_{2,3}(q, \varepsilon)$ can be similarly analyzed using integration by parts, which gives

$$
\varepsilon^{2 m-4} \ln \varepsilon \int_{3 \varepsilon}^{1} \ln q \tilde{H}_{2,3}(q, \varepsilon) e^{-q x} d q=-\varepsilon^{2 m-4} \ln \varepsilon \tilde{H}_{2,3}(1, \varepsilon) e^{-x}+R_{s}(x, \varepsilon),
$$


where $R_{s}$ satisfies (37). The term containing $(\ln q)^{2} \tilde{H}_{2,3}(q, \varepsilon)$ satisfies (37) by direct calculation and counting powers of $\varepsilon$.

Finally we show that $S_{3}(x, \varepsilon)$ satisfies the estimate (37). Denoting $\partial_{(i, j)} \tilde{R}(u, v)=$ $\partial^{i+j} \tilde{R}(u, v) /(\partial u)^{i}(\partial v)^{j}$, we have by Lemma 2

$$
\begin{array}{r}
\left|\frac{\partial^{k}}{\partial \varepsilon^{k}}\left(\varepsilon \int_{3 \varepsilon}^{1} \frac{\tilde{R}(q / \varepsilon, q) e^{-q x}}{q(q+2 \varepsilon)} d q\right)\right|=\left|\frac{\partial^{k}}{\partial \varepsilon^{k}} \int_{3}^{1 / \varepsilon} \frac{\tilde{R}(\tau, \varepsilon \tau) e^{-\varepsilon \tau x}}{\tau(\tau+2)} d \tau\right| \\
\lesssim \sum_{i+j=k}\left|\int_{3}^{1 / \varepsilon} \frac{\partial_{(0, i)} \tilde{R}(\tau, \varepsilon \tau) \tau^{i-1} x^{j} e^{-\varepsilon \tau x}}{\tau+2} d \tau\right|+\sum_{i+j=k-1}\left|\frac{\partial_{(i, 0)} \tilde{R}(1 / \varepsilon, 1) e^{-x}}{\varepsilon^{2 i+j}}\right| \\
\lesssim(|\ln \varepsilon|+1)^{4}\left(\left|\varepsilon^{1-k}\right| e^{-x}+\sum_{i+j=k}\left|\varepsilon^{1-i} x^{j}\right|\right)
\end{array}
$$

for $0 \leqslant k \leqslant m+1$. Thus

$$
\begin{aligned}
\left|\frac{\partial^{n} S_{3}(x, \varepsilon)}{\partial \varepsilon^{n}}\right| & \lesssim \sum_{j+k=n}\left|\varepsilon^{m-1-j} \frac{\partial^{k}}{\partial \varepsilon^{k}}\left(\varepsilon \int_{3 \varepsilon}^{1} \frac{\tilde{R}(q / \varepsilon, q) e^{-q x}}{q(q+2 \varepsilon)} d q\right)\right| \\
& \lesssim\left|(|\ln \varepsilon|+1)^{4} \varepsilon^{m-n}\right| .
\end{aligned}
$$

Finally $s^{\prime}$ and $s^{\prime \prime}$ are similarly analyzed, finishing the proof of Lemma 4 .

4.3. Detailed behavior of the exponentially decaying functions $y_{ \pm}$. Since $\hat{\psi}$ solves (2), its singularity structure depends on the singularity structure of the two solutions of the associated homogeneous equation (3), which are of the form $y_{ \pm}(x)=e^{\mp \varepsilon x}\left(1+s_{ \pm}(x)\right)$ where $s_{ \pm}(x)=o(1)$ for $x \rightarrow \pm \infty$. By symmetry it is sufficient to study $y_{+}(x)$, where $s_{+}$is exactly the function $s$ in Lemma 4 .

Proposition 7 below shows that $y_{+}(x)$ has an expansion involving the special functions

$$
\begin{gathered}
\Phi_{1}(x)=(m-2)^{1 /(m-2)} \Gamma\left(\frac{m-1}{m-2}\right) \sqrt{x} I_{\frac{1}{m-2}}\left(\frac{2 x^{1-m / 2}}{m-2}\right), \\
\Phi_{2}(x)=\frac{(m-2)^{-1 /(m-2)}}{\Gamma\left(\frac{1}{m-2}\right)} \sqrt{x} K_{\frac{1}{m-2}}\left(\frac{2 x^{1-m / 2}}{m-2}\right),
\end{gathered}
$$

where $I_{n}$ and $K_{n}$ denote the modified Bessel functions of the first and second kind respectively.

Proposition 7. For arbitrarily small $\delta>0, x \geqslant x_{+}$and $|\varepsilon| \leqslant 1 / x$ we have

$$
y_{+}(x ; \varepsilon)=r(\varepsilon)\left(\Phi_{1}(x)+B_{1}(x) \varepsilon^{m-1} \ln \varepsilon+\hat{f}_{a}(x, \varepsilon)\right),
$$

where

$$
r(\varepsilon):=\left(1+a_{1} \varepsilon^{m-2} \ln \varepsilon+a_{0} \varepsilon^{2 m-4}(\ln \varepsilon)^{2}\right),
$$

where $a_{0,1}$ are constants, $B_{1}(x)$ is a linear combination of $\Phi_{1,2}(x)$ with $B_{1}(x) \sim$ const. $x, \hat{f}_{a}(x, \varepsilon) \lesssim|\varepsilon x|$, and

$$
\sup _{1 \leqslant k \leqslant m-1}\left|x^{-k} \frac{\partial^{k} \hat{f}_{a}(x ; \varepsilon)}{\partial \varepsilon^{k}}\right|<\infty ; \sup _{m \leqslant k \leqslant m+1}\left|\varepsilon^{k-1-m+\delta} x^{-m-1} \frac{\partial^{k} \hat{f}_{a}(x ; \varepsilon)}{\partial \varepsilon^{k}}\right|<\infty .
$$


Furthermore, the expansion (55) is differentiable in $x$; i.e., (57) holds with $\hat{f}_{a}(x ; \varepsilon)$ replaced by $x \hat{f}_{a}^{\prime}(x ; \varepsilon)$.

Proof. It follows from Lemma 4 that $y_{+}=e^{-\varepsilon x}(1+s)$ has the following expansion:

$$
\begin{aligned}
y_{+}(x ; \varepsilon)= & h_{0}(x)+h_{1}(x) \varepsilon+h_{2}(x) \varepsilon^{m-2} \ln \varepsilon+h_{3}(x) \varepsilon^{m-1} \ln \varepsilon \\
& +h_{4}(x) \varepsilon^{2 m-4}(\ln \varepsilon)^{2}+\tilde{H}(x ; \varepsilon),
\end{aligned}
$$

where $h_{0} \sim 1, h_{2} \sim a_{1}, h_{4} \sim a_{0}$ for large $x$, and $\tilde{H}(x ; \varepsilon)$ satisfies (57) with $|\tilde{H}(x ; \varepsilon)| \lesssim\left|\varepsilon^{2} x^{2}\right|$. Plugging this expansion back into (3) (recall that the asymptotics (58) is differentiable by Lemma 4) we have

$$
\begin{aligned}
0= & \left(h_{0}^{\prime \prime}(x)-\frac{1}{x^{m}} h_{0}(x)\right)+\left(h_{1}^{\prime \prime}(x)-\frac{1}{x^{m}} h_{1}(x)\right) \varepsilon \\
& +\left(h_{2}^{\prime \prime}(x)-\frac{1}{x^{m}} h_{2}(x)\right) \varepsilon^{m-2} \ln \varepsilon+\ldots
\end{aligned}
$$

Standard asymptotic arguments for $\varepsilon \rightarrow 0$ show that all the coefficients above must be zero, and thus $h_{i}$ satisfies the equation

$$
f^{\prime \prime}(x)=x^{-m} f(x) .
$$

The solutions to (60) are exactly $\Phi_{1,2}$. Note that $\Phi_{1}(x)=1+o(1)$ and $\Phi_{2}(x)=$ $x(1+o(1))$ for large $x$. Therefore $h_{0}(x)=\Phi_{1}(x), h_{2}(x)=a_{1} \Phi_{1}(x)$, and $h_{4}(x)=$ $a_{0} \Phi_{1}(x)$ since $h_{0} \sim 1, h_{2} \sim a_{1}$, and $h_{4} \sim a_{0}$.

Thus dividing (58) by (56) we obtain (55) for some $B_{1}$. Substituting (55) into (13) and examining the coefficients of $\varepsilon^{m-1} \ln \varepsilon$ we see that $B_{1}$ satisfies (60) and is thus a linear combination of $\Phi_{1,2}$. The large $x$ behavior of $B_{1}$ follows from (35) and (36). Differentiability of (55) follows from the last paragraph of Lemma 4 .

Note 4. (i) A similar conclusion is true for $y_{-}$.

(ii) Without loss of generality we can assume $r(\varepsilon) \neq 0$, since otherwise we can modify the definition of $r(\varepsilon)$ by adding $\varepsilon$ to it.

4.4. Estimating the exponentially decaying functions $y_{ \pm}$for large $\varepsilon$. To prove the main results we use Proposition 7 and the properties of the inverse Laplace transform of $y_{ \pm}(x ; \varepsilon)$ (see Section [5). These properties depend on the behavior of $y_{ \pm}$and $s_{ \pm}$together with its $\varepsilon$ derivatives in $\mathbb{H}$ for large $\varepsilon$. Let $\mathcal{D}=\left(x_{+}, \infty\right) \times \overline{\mathbb{H}}$. First we prove the following lemma.

Lemma 8. Assume $\left|V^{(k)}(x)\right| \lesssim x^{-m-k}$ for $x \geqslant x_{+}$and $0 \leqslant k \leqslant 2$, and $s(x ; \varepsilon)=$ $s_{+}(x ; \varepsilon)$ solves (7) with $s(x ; \varepsilon)=o(1)$ for $x \rightarrow \infty$. Then for any fixed $x_{1} \in \mathbb{R}$ the function $s(x ; \cdot)$ is analytic in $\mathbb{H}$ and continuous in $\overline{\mathbb{H}}$ for $x \geqslant x_{1}$. Moreover, we have

$$
|s(x ; \varepsilon)| \lesssim(|\varepsilon|\langle x\rangle+1)^{-1}\langle x\rangle^{-m+2} ; \quad\left|s^{\prime}(x ; \varepsilon)\right| \lesssim(|\varepsilon|\langle x\rangle+1)^{-1}\langle x\rangle^{-m+1}
$$

uniformly in $\mathcal{D}$, and for $|\varepsilon| \geqslant 1 /\langle x\rangle$ we have

$$
\left|\frac{\partial^{n} s(x ; \varepsilon)}{\partial \varepsilon^{n}}\right| \lesssim|\varepsilon|^{-1-n}\langle x\rangle^{1-m} ;\left|\frac{\partial^{n} s^{\prime}(x ; \varepsilon)}{\partial \varepsilon^{n}}\right| \lesssim|\varepsilon|^{-1-n}\langle x\rangle^{-m} ; 0 \leqslant n \leqslant m+1 .
$$

A similar conclusion is true for $s_{-}$; i.e., (61) and (62) hold for both $s_{+}$and $s_{-}$. 
Proof. We write (7) in integral form:

$$
\begin{aligned}
s(x) & =\int_{\infty}^{x} \int_{\infty}^{t} e^{-2 \varepsilon\left(t^{\prime}-t\right)} V\left(t^{\prime}\right) d t^{\prime} d t+\int_{\infty}^{x} \int_{\infty}^{t} e^{-2 \varepsilon\left(t^{\prime}-t\right)} V\left(t^{\prime}\right) s\left(t^{\prime}\right) d t^{\prime} d t \\
& :=T_{1}(x ; V)+L s .
\end{aligned}
$$

It is straightforward to check that $\left|T_{1}(x ; V)\right| \lesssim x^{2-m}$, and by integration by parts,

$$
\left|T_{1}(x ; V)\right| \leqslant \frac{1}{2|\varepsilon|}\left|\int_{\infty}^{x}\left(V(t)-\int_{\infty}^{t} e^{-2 \varepsilon\left(t^{\prime}-t\right)} V^{\prime}\left(t^{\prime}\right) d t^{\prime}\right) d t\right| \lesssim|\varepsilon|^{-1} x^{1-m} .
$$

Thus

$$
\left|T_{1}(x ; V)\right| \lesssim \min \left(x^{2-m},|\varepsilon|^{-1} x^{1-m}\right) \lesssim(|\varepsilon x|+1)^{-1} x^{2-m}
$$

uniformly in $x \geqslant x_{+}$and $\varepsilon \in \overline{\mathbb{H}}$. We analyze (63) in the Banach space $\mathcal{B}$ of functions $f: \mathcal{D} \rightarrow \mathbb{C}$ such that $f(x, \cdot)$ is analytic in $\mathbb{H}$, continuous in $\overline{\mathbb{H}}$, with the norm

$$
\|s\|=\sup _{(x, \varepsilon) \in \mathcal{D}}|s(x, \varepsilon)|<\infty .
$$

We see that $T_{1} \in \mathcal{B}$ and

$$
\|L\| \leqslant\left|\int_{\infty}^{x} \int_{\infty}^{t} V\left(t^{\prime}\right) d t^{\prime} d t\right| \leqslant \text { const. } x^{2-m} .
$$

Thus if $x_{0}$ is sufficiently large, then (63) is contractive in $\mathcal{B}$ and has a unique solution for $x \geqslant x_{0}$. Then, the first estimate in (61) is obtained by taking $x_{0}$ sufficiently large and writing

$$
|s(x, \varepsilon)| \leqslant\left|(1-L)^{-1} T_{1}\right| .
$$

We see by direct differentiation of the rhs of (63) that

$$
s^{\prime}(x ; \varepsilon)=\int_{\infty}^{x} e^{-2 \varepsilon\left(t^{\prime}-x\right)} V\left(t^{\prime}\right) d t^{\prime}+\int_{\infty}^{x} e^{-2 \varepsilon\left(t^{\prime}-x\right)} V\left(t^{\prime}\right) s\left(t^{\prime}\right) d t^{\prime} .
$$

This implies

$$
\left|s^{\prime}(x ; \varepsilon)\right| \lesssim x^{1-m},
$$

and by integration by parts and the first estimate in (61),

$$
\left|s^{\prime}(x, \varepsilon)+\frac{1}{2 \varepsilon}\left(V(x)-\int_{\infty}^{x} e^{-2 \varepsilon\left(t^{\prime}-x\right)} V^{\prime}\left(t^{\prime}\right) d t^{\prime}\right)\right| \lesssim|\varepsilon|^{-1} x^{2(1-m)} .
$$

Thus

$$
\left|s^{\prime}(x, \varepsilon)\right| \lesssim \min \left(|\varepsilon|^{-1} x^{-m}, x^{1-m}\right) \lesssim(|\varepsilon x|+1)^{-1} x^{1-m}
$$

Rewriting (69) as

$$
s^{\prime}(x ; \varepsilon)=\int_{\infty}^{0} e^{-2 \varepsilon t} V(t+x) d t+\int_{\infty}^{0} e^{-2 \varepsilon t} V(t+x) s(t+x) d t,
$$

we have by integration by parts

$$
s^{\prime \prime}(x ; \varepsilon)=-\frac{1}{2 \varepsilon}\left(V^{\prime}(x)-\int_{\infty}^{0} e^{-2 \varepsilon t} V^{\prime \prime}(t+x) d t\right)+\int_{\infty}^{0} e^{-2 \varepsilon t}(V(t+x) s(t+x))^{\prime} d t,
$$

which implies

$$
\left|s^{\prime \prime}(x ; \varepsilon)\right| \lesssim|\varepsilon|^{-1} x^{-m-1} .
$$


For the $\varepsilon$ derivatives, the proof is by induction on $n$. The equation for $u=\frac{d}{d \varepsilon} s(x, \varepsilon)$ is

$$
u^{\prime \prime}-2 \varepsilon u^{\prime}-V(x) u=2 s^{\prime}(x) .
$$

By (73) we have

$$
u(x)=T_{1}\left(x ; 2 s^{\prime}\right)+L u
$$

(see (63)). Using (71) and (72) we obtain

$$
\left|T_{1}\left(x ; 2 s^{\prime}\right)\right| \leqslant \frac{1}{|\varepsilon|}\left|\int_{\infty}^{x}\left(s^{\prime}(t)-\int_{\infty}^{t} e^{-2 \varepsilon\left(t^{\prime}-t\right)} s^{\prime \prime}\left(t^{\prime}\right) d t^{\prime}\right) d t\right| \lesssim|\varepsilon|^{-2} x^{1-m} .
$$

Thus (67) and (74) imply

$$
\begin{gathered}
|u(x, \varepsilon)| \lesssim|\varepsilon|^{-2} x^{1-m}, \\
\left|u^{\prime}(x, \varepsilon)\right|=\left|2 \int_{\infty}^{x} e^{-2 \varepsilon\left(t^{\prime}-x\right)} s^{\prime}\left(t^{\prime}\right) d t^{\prime}+\int_{\infty}^{x} e^{-2 \varepsilon\left(t^{\prime}-x\right)} V\left(t^{\prime}\right) u\left(t^{\prime}\right) d t^{\prime}\right| \lesssim|\varepsilon|^{-2} x^{-m} .
\end{gathered}
$$

Taking $k \varepsilon$-derivatives of (73) and letting $u_{k}(x, \varepsilon)=\partial^{k} s(x, \varepsilon) / \partial \varepsilon^{k}$ we have

$$
u_{k}^{\prime \prime}-2 \varepsilon u_{k}^{\prime}-V(x) u_{k}=2 k u_{k-1}^{\prime},
$$

which gives by induction

$$
\begin{gathered}
\left|u_{k}(x, \varepsilon)\right| \leqslant\left|(1-L)^{-1} T_{1}\left(x ; 2 k u_{k-1}^{\prime}\right)\right| \lesssim|\varepsilon|^{-1-k}|x|^{1-m}, \\
\left|u_{k}^{\prime}(x, \varepsilon)\right| \lesssim|\varepsilon|^{-1-k}|x|^{-m} .
\end{gathered}
$$

Finally, for $x_{1} \leqslant x \leqslant x_{0}$ existence and analyticity of the solution follow from standard analytic dependence on parameters. Thus (61) and (62) only need to be verified for large $\varepsilon$ since the $x$ dependence is irrelevant. Note that by (17) we have

$$
s(x)=\frac{1}{2 \varepsilon} \int_{\infty}^{x}\left(e^{-2 \varepsilon(t-x)}-1\right) V(t)(1+s(t)) d t,
$$

which is contractive for large $\varepsilon$ implying $|s(x)| \lesssim 1 /|\varepsilon|$. The result for $s^{\prime}$ then follows from (69). Similarly, using (76) we see that

$$
u_{k}(x)=\frac{1}{\varepsilon} \int_{\infty}^{x}\left(e^{-2 \varepsilon(t-x)}-1\right)\left(2 k u_{k-1}^{\prime}(t)+V(t) u_{k}(t)\right) d t,
$$

$$
u_{k}^{\prime}(x)=\frac{1}{\varepsilon}\left(2 k u_{k-1}^{\prime}(x)+V(x) u_{k}(x)-\int_{\infty}^{x} e^{-2 \varepsilon(t-x)}\left(2 k u_{k-1}^{\prime}(t)+V(t) u_{k}(t)\right)^{\prime} d t\right),
$$

which gives (62) by induction.

Proposition 7 and Lemma 8 apply to the cases $|\varepsilon| \leqslant 1 /\langle x\rangle$ and $|\varepsilon| \geqslant 1 /\langle x\rangle$ respectively, but it is more convenient to have an expansion for $|\varepsilon| \leqslant 1$. This is established in the following.

Corollary 9. For $\pm\left(x-x_{\mp}\right) \geqslant 0$ and $|\varepsilon| \leqslant 1$, the exponentially decaying solution $y_{ \pm}$satisfies

$$
y_{ \pm}(x)=r_{ \pm}(\varepsilon) e^{\mp \varepsilon x}\left(\Phi_{1}^{ \pm}(x)+\frac{B_{2}^{ \pm}(x) \varepsilon^{m-1} \ln \varepsilon}{1+\varepsilon\langle x\rangle}+R_{f}^{ \pm}(x ; \varepsilon)\right),
$$


where $r_{ \pm}(\varepsilon)$ are of the form (56), the modified Bessel functions $\tilde{\Phi}_{1}^{ \pm}(x) \sim 1$ and $B_{2}^{ \pm}(x) \sim$ const. $x$ for $x \rightarrow \pm \infty$, and (57) holds with $R_{f}^{ \pm}(x ; \varepsilon)$ replacing $\hat{f}_{a}$. Also

$$
y_{ \pm}^{\prime}(x)=r_{ \pm}(\varepsilon) e^{\mp \varepsilon x}\left(\Phi_{1}^{\prime \pm}(x)+\frac{B_{2}^{\prime} \pm(x) \varepsilon^{m-1} \ln \varepsilon}{1+\varepsilon\langle x\rangle}+r_{f}^{ \pm}(x ; \varepsilon)\right)
$$

where (57) holds with $\langle x\rangle r_{f}^{\prime \pm}(x)$ replacing $\hat{f}_{a}$.

Proof. Without loss of generality we show the result for $y_{+}$. By Proposition 7 we see that (80) holds for $|\varepsilon| \leqslant 1 / x$, since multiplying by $e^{\varepsilon x}$ and $1 /(1+\varepsilon\langle x\rangle)$ does not change the structure of (55). For $1 / x \leqslant|\varepsilon| \leqslant 1$, we only need to show that (57) is valid with $\hat{f}_{a}$ replaced by $R_{f}(x ; \varepsilon)$. By (62) we have for $1 / x \leqslant|\varepsilon| \leqslant 1$

$$
\left|\frac{\partial^{n} s_{+}(x ; \varepsilon)}{\partial \varepsilon^{n}}\right| \lesssim x^{n+2-m} ; 0 \leqslant n \leqslant m+1 .
$$

Now $\left(r_{+}(\varepsilon)\right)^{-1}\left(1+s_{+}(x ; \varepsilon)\right)$ satisfies the estimates for $\hat{f}_{a}$ in (57) by (82) (cf. Note 44). Since $\frac{B_{2}^{+}(x) \varepsilon^{m-1} \ln \varepsilon}{1+\varepsilon\langle x\rangle}$ obviously satisfies the estimates in (157), we see that $R_{f}$ satisfies (57). Differentiability of (80) follows from Lemma 4 and Lemma 8 .

\section{Singularity PROPERTies OF $\hat{\psi}$ FOR $x \in \mathbb{R}$}

Corollary 9 gives the needed properties of $y_{ \pm}$for $\pm\left(x-x_{\mp}\right) \geqslant 0$. Now we extend the results to all $x \in \mathbb{R}$.

5.1. Exponentially decaying functions $y_{ \pm}$on the real line. Assume $V(x)=$ $v_{1} x^{-m}$ for $x \geqslant x_{+}$and $V(x)=v_{2} x^{-m}$ for $x \leqslant x_{-}$. We now show the following.

Proposition 10. The exponentially decaying functions $y_{ \pm}$are analytic in $\varepsilon \in \mathbb{H}$ for all $x \in \mathbb{R}$. Moreover, for $|\varepsilon| \leqslant 1$ they satisfy

$$
y_{ \pm}(x)=r_{ \pm}(\varepsilon) e^{\mp \varepsilon x}\left(r_{0}^{ \pm}(x)+\frac{r_{1}^{ \pm}(x) \varepsilon^{m-1} \ln \varepsilon}{1+\varepsilon\langle x\rangle}+R_{0}^{ \pm}(x ; \varepsilon)\right),
$$

where $\left|r_{0}^{ \pm}(x)\right| \lesssim\langle x\rangle$ and $\left|r_{1}^{ \pm}(x)\right| \lesssim\langle x\rangle^{2}$ for all $x \in \mathbb{R}$, and for $\pm x \geqslant \pm x_{ \pm}, r_{0}^{ \pm}(x)$ and $r_{1}^{ \pm}(x)$ are explicit modified Bessel functions with $\left|r_{0}^{ \pm}(x)\right| \lesssim 1,\left|r_{1}^{ \pm}(x)\right| \lesssim\langle x\rangle$ (cf. Corollary 9). Furthermore $R_{0}^{ \pm}(x ; 0)=0$, and for arbitrarily small $\delta>0$ we have

$$
\begin{aligned}
& \sup _{\substack{0 \leqslant k \leqslant m-1 \\
\pm\left(x-x_{\mp}\right) \geqslant 0}}\left|\langle x\rangle^{-k} \frac{\partial^{k} R_{0}^{ \pm}(x ; \varepsilon)}{\partial \varepsilon^{k}}\right| \lesssim 1 ; \sup _{\substack{m \leqslant k \leqslant m+1 \\
\pm\left(x-x_{\mp}\right) \geqslant 0}}|\varepsilon|^{k-m+\delta}\langle x\rangle^{-m} \frac{\partial^{k} R_{0}^{ \pm}(x ; \varepsilon)}{\partial \varepsilon^{k}} \mid \lesssim 1, \\
& \sup _{\substack{0 \leqslant k \leqslant m-1 \\
\pm\left(x-x_{\mp}\right) \leqslant 0}}\left|\langle x\rangle^{-k-1} \frac{\partial^{k} R_{0}^{ \pm}(x ; \varepsilon)}{\partial \varepsilon^{k}}\right| \lesssim 1 ;\left.\sup _{\substack{m \leqslant k \leqslant m+1 \\
\pm\left(x-x_{\mp}\right) \leqslant 0}}|| \varepsilon\right|^{k-m+\delta}\langle x\rangle^{-m-1} \frac{\partial^{k} R_{0}^{ \pm}(x ; \varepsilon)}{\partial \varepsilon^{k}} \mid \lesssim 1 .
\end{aligned}
$$

In addition, (83) is differentiable in $x$; i.e., (84) holds with $R_{0}^{ \pm}(x ; \varepsilon)$ replaced with $\langle x\rangle R_{0}^{\prime \pm}(x ; \varepsilon)$.

For $|\varepsilon| \geqslant 1$ we have

$$
y_{ \pm}(x ; \varepsilon)=e^{\mp \varepsilon x}\left(1+s_{ \pm}(x ; \varepsilon)\right),
$$


where $s_{ \pm}(x ; \varepsilon)$ satisfies (62) for $\pm\left(x-x_{\mp}\right) \geqslant 0$ and

$$
\left|\frac{\partial^{n} s_{ \pm}(x ; \varepsilon)}{\partial \varepsilon^{n}}\right| \lesssim|\varepsilon|^{-1}\langle x\rangle^{n} ;\left|\frac{\partial^{n} s_{ \pm}^{\prime}(x ; \varepsilon)}{\partial \varepsilon^{n}}\right| \lesssim|\varepsilon|^{-1}\langle x\rangle^{n} ; 0 \leqslant n \leqslant m+1,
$$

for $\pm\left(x-x_{\mp}\right) \leqslant 0$.

Proof. We only analyze $y_{+}$since $y_{-}$is similar. For $x \geqslant x_{+}$, the behavior of $y_{+}$for small $\varepsilon$ is given in Corollary 9 and the behavior for large $\varepsilon$ is given in Lemma 8 ,

First assume $|\varepsilon| \leqslant 1$. In the middle region $x_{-} \leqslant x \leqslant x_{+}$there exist two linearly independent solutions analytic in $\varepsilon$ (by analytic dependence on parameters of ODE), and the $x$ bounds are obvious. Clearly $y_{+}$is a linear combination of these two solutions, and thus the analytic structure of $y_{+}$is preserved (cf. Corollary 9). For $x \leqslant x_{-}$, by (61) we can assume $x_{-}$is large enough such that $y_{-}\left(x_{-}\right) \neq 0$. By standard ODE results $y_{+}$satisfies

$$
y_{+}(x ; \varepsilon)=y_{-}(x ; \varepsilon)\left(\frac{y_{+}\left(x_{-} ; \varepsilon\right)}{y_{-}\left(x_{-} ; \varepsilon\right)}-W(\varepsilon) \int_{x_{-}}^{x} \frac{1}{y_{-}^{2}(t ; \varepsilon)} d t\right),
$$

where $W(\varepsilon)=y_{+} y_{-}^{\prime}-y_{+}^{\prime} y_{-}$is the Wronskian.

One can verify by direct calculation that $y_{+}$solves (3) for $x \leqslant x_{-}$and is differentiable at $x_{-}$. By Corollary 9 and the reasoning above we see that for $x_{-} \leqslant x \leqslant x_{+}$,

$$
y_{+}(x ; \varepsilon)=r(\varepsilon) e^{-\varepsilon x}\left(\Phi_{0}(x)+\frac{\hat{B}_{2}(x) \varepsilon^{m-1} \ln \varepsilon}{1+\varepsilon\langle x\rangle}+\hat{R}_{f}(x ; \varepsilon)\right),
$$

where $\Phi_{0}$ and $\hat{B}_{2}$ are smooth, and where (57) holds with $\hat{R}_{f}$ replacing $\hat{f}_{a}$.

Note 5. Assume $|\varepsilon| \leqslant 1$. Since the Wronskian $W$ is independent of $x$, by direct calculation using (88) and (80) at $x_{-}$for $|\varepsilon| \leqslant 1$, we have

$$
W(\varepsilon)=r_{+}(\varepsilon) r_{-}(\varepsilon)\left(q_{1} \varepsilon^{m-1} \ln \varepsilon+q_{2}(\varepsilon)\right),
$$

where $q_{1}$ is a constant, and by Corollary 9 we have that $\frac{d^{k} q_{2}(\varepsilon)}{d \varepsilon^{k}}$ is bounded for $0 \leqslant k \leqslant m-1$ and that $\varepsilon^{k-m+\delta} \frac{d^{k} q_{2}(\varepsilon)}{d \varepsilon^{k}}$ is bounded for $m \leqslant k \leqslant m+1$ and arbitrarily small $\delta>0$. In the absence of zero-energy resonance, $y_{+}$and $y_{-}$are linearly independent for small $\varepsilon$, and thus $q_{2}(0)=W(0) \neq 0$.

Assume $|\varepsilon| \geqslant 1$. By direct calculation using (85) at $x_{-}$for $|\varepsilon| \geqslant 1$, denoting

$$
q_{3}(\varepsilon)=W(\varepsilon)-2 \varepsilon
$$

we have that $\frac{d^{k} q_{3}(\varepsilon)}{d \varepsilon^{k}}$ is bounded for $0 \leqslant k \leqslant m+1$.

By (87) we have

$$
e^{\varepsilon x} y_{+}(x ; \varepsilon)=r(\varepsilon)\left(\hat{\Phi}_{1}(x)+\frac{B_{3}(x) \varepsilon^{m-1} \ln \varepsilon}{1+\varepsilon\langle x\rangle}+x \hat{f}_{b}(x ; \varepsilon)\right),
$$

where $\hat{\Phi}_{1}(x)=\Phi_{1}^{-}(x)\left(\frac{\Phi_{1}^{+}\left(x_{-}\right)}{\Phi_{1}^{-}\left(x_{-}\right)}-W(0) \int_{x_{-}}^{x} \frac{1}{\Phi_{1}^{-2}(t)} d t\right)$ satisfies $\left|\hat{\Phi}_{1}(x)\right| \lesssim\langle x\rangle, B_{3}$ is a smooth function with $\left|B_{3}\right| \lesssim\langle x\rangle^{2}$ and (57) holds with $\hat{f}_{a}$ replaced by $\hat{f}_{b}$ by 
(87), which can be checked using the fact that for any bounded function $f$ we have

$$
\left|e^{2 \varepsilon x} \int_{x_{-}}^{x} e^{-2 \varepsilon t} t^{k} f(t) d t\right| \lesssim|x|^{k+1}
$$

Thus (83) is valid for $x \leqslant x_{-}$.

Assume $|\varepsilon| \geqslant 1$. In the middle region $x_{-} \leqslant x \leqslant x_{+}$the result [85) follows from Lemma 8

For $x \leqslant x_{-}$, we denote $f_{ \pm}=1+s_{ \pm}$, and by (87) and Lemma 8 we have

$$
f_{+}(x ; \varepsilon)=e^{\varepsilon x} y_{+}(x ; \varepsilon)=f_{-}(x ; \varepsilon)\left(1+s_{3}(x ; \varepsilon)\right),
$$

where

$$
\begin{aligned}
s_{3}(x ; \varepsilon)= & -1+\frac{f_{+}\left(x_{-} ; \varepsilon\right)}{f_{-}\left(x_{-} ; \varepsilon\right)} e^{2 \varepsilon\left(x-x_{-}\right)}-\left(2 \varepsilon+q_{3}(\varepsilon)\right) \int_{x_{-}}^{x} \frac{e^{2 \varepsilon(x-t)}}{f_{-}^{2}(t ; \varepsilon)} d t \\
= & \frac{s_{+}\left(x_{-} ; \varepsilon\right)-s_{-}\left(x_{-} ; \varepsilon\right)}{f_{-}\left(x_{-} ; \varepsilon\right)} e^{2 \varepsilon\left(x-x_{-}\right)}-q_{3}(\varepsilon) \int_{x_{-}}^{x} \frac{e^{2 \varepsilon(x-t)}}{f_{-}^{2}(t ; \varepsilon)} d t \\
& +2 \varepsilon \int_{x_{-}}^{x} \frac{e^{2 \varepsilon(x-t)} s_{-}(t ; \varepsilon)\left(s_{-}(t ; \varepsilon)+2\right)}{f_{-}^{2}(t ; \varepsilon)} d t .
\end{aligned}
$$

Using (62) in Lemma 8 we see that the first term in (92) satisfies the estimates in (86). By integration by parts we obtain

$$
q_{3}(\varepsilon) \int_{x_{-}}^{x} \frac{e^{2 \varepsilon(x-t)}}{f_{-}^{2}(t ; \varepsilon)} d t=\frac{q_{3}(\varepsilon)}{2 \varepsilon}\left(\frac{e^{2 \varepsilon\left(x-x_{-}\right)}}{f_{-}^{2}\left(x_{-} ; \varepsilon\right)}-\frac{1}{f_{-}^{2}(x ; \varepsilon)}+\int_{x_{-}}^{x} e^{2 \varepsilon(x-t)}\left(\frac{1}{f_{-}^{2}(t ; \varepsilon)}\right)^{\prime} d t\right)
$$

and

$$
\begin{aligned}
2 \varepsilon \int_{x_{-}}^{x} & \frac{e^{2 \varepsilon(x-t)} s_{-}(t ; \varepsilon)\left(s_{-}(t ; \varepsilon)+2\right)}{f_{-}^{2}(t ; \varepsilon)} d t=\frac{e^{2 \varepsilon\left(x-x_{-}\right)} s_{-}\left(x_{-} ; \varepsilon\right)\left(s_{-}\left(x_{-} ; \varepsilon\right)+2\right)}{f_{-}^{2}\left(x_{-} ; \varepsilon\right)} \\
& -\frac{s_{-}(x ; \varepsilon)\left(s_{-}(x ; \varepsilon)+2\right)}{f_{-}^{2}(x ; \varepsilon)}+\int_{x_{-}}^{x} e^{2 \varepsilon(x-t)}\left(\frac{s_{-}(t ; \varepsilon)\left(s_{-}(t ; \varepsilon)+2\right)}{f_{-}^{2}(t ; \varepsilon)}\right)^{\prime} d t
\end{aligned}
$$

Thus using the estimates in Lemma 8 as well as (92) we see that the first estimate in (86) holds with $s_{+}$replaced by $s_{3}$.

To show (86) for $s_{+}$, note that by (91) we have $s_{+}(x ; \varepsilon)=s_{-}(x ; \varepsilon)+s_{3}(x ; \varepsilon)-$ $s_{-}(x ; \varepsilon) s_{3}(x ; \varepsilon)$. Since $s_{-}$satisfies (62) and $s_{3}$ satisfies $\left|\frac{\partial^{n} s_{3}(x ; \varepsilon)}{\partial \varepsilon^{n}}\right| \lesssim|\varepsilon|^{-1}\langle x\rangle^{n}$, we see that the first estimate in (86) holds for $s_{+}$.

To estimate $s_{+}^{\prime}$, note that by (87) and (91)

$$
\begin{aligned}
& s_{+}^{\prime}(x ; \varepsilon)=\left(s_{-}^{\prime}(x ; \varepsilon)+2 \varepsilon+2 \varepsilon s_{-}(x ; \varepsilon)\right)\left(1+s_{3}(x ; \varepsilon)\right)-\frac{W(\varepsilon)}{h_{-}(x ; \varepsilon)} \\
= & 2 \varepsilon\left(s_{-}(x ; \varepsilon)+s_{3}(x ; \varepsilon)-s_{+}(x ; \varepsilon)\right)+O\left(\varepsilon^{-1}\right)=2 \varepsilon s_{-}(x ; \varepsilon) s_{3}(x ; \varepsilon)+O\left(\varepsilon^{-1}\right) .
\end{aligned}
$$

Thus the second inequality of (86) holds.

The proof for $s_{-}$is similar by symmetry. 
5.2. Analysis of $\hat{\psi}$. Recall that the solution of (2) is given in (5). Note that $W(\varepsilon) \neq 0$ for $\varepsilon \in \overline{\mathbb{H}}$ by the assumption of no bound state and no zero energy resonance.

To obtain the estimates for $\frac{\sin (t \sqrt{A})}{\sqrt{A}} \psi_{1}$ and $\cos (t \sqrt{A}) \psi_{0}$ in Theorem 1, we need the $\varepsilon$-expansions of $\mathcal{G}\left(\psi_{1}\right)$ and $\mathcal{G}\left(\varepsilon \psi_{0}\right)$ :

Proposition 11. For $\varepsilon \in \overline{\mathbb{H}} \backslash\{0\}$ we have

$$
\mathcal{G}\left(\psi_{1}\right)=r_{3}(x) \frac{\varepsilon^{m-1} \ln \varepsilon}{(1+\varepsilon\langle x\rangle)^{m+2}}+\mathcal{G}_{0}\left(\psi_{1}(x)\right)+R(x ; \varepsilon),
$$

where

$$
\begin{gathered}
\left\|\langle x\rangle^{-2} r_{3}(x)\right\|_{\infty} \lesssim\left\|\langle x\rangle^{2} \psi_{1}(x)\right\|_{1}, \\
\mathcal{G}_{0}(\psi):=\frac{1}{2(1+\varepsilon)}\left(\int_{\infty}^{x} e^{-\varepsilon(u-x)} \psi(u) d u-\int_{-\infty}^{x} e^{\varepsilon(u-x)} \psi(u) d u\right), \\
\left\|\langle x\rangle^{-k-2} \frac{\partial^{k}}{\partial \varepsilon^{k}} R(x, \varepsilon)\right\|_{\infty} \lesssim(1+|\varepsilon|)^{-2}\left\|\langle x\rangle^{k+2} \psi_{1}(x)\right\|_{1} \quad(0 \leqslant k \leqslant m-1), \\
\left\|\langle x\rangle^{-m-2} \frac{\partial^{m}}{\partial \varepsilon^{m}} R(x, \varepsilon)\right\|_{\infty} \lesssim\left(|\varepsilon|^{\delta}+|\varepsilon|\right)^{-2}\left\|\langle x\rangle^{m+2} \psi_{1}(x)\right\|_{1}
\end{gathered}
$$

for arbitrary $\delta>0$.

Proof. The fact that $\mathcal{G}\left(\psi_{1}\right)$ is analytic in $\varepsilon \in \mathbb{H}$ follows easily from Proposition 10,

We first consider the case $|\varepsilon| \leqslant 1$. By (83) we have

$$
y_{-}(x) \int_{\infty}^{x} y_{+}(u) \psi_{1}(u) d u-y_{+}(x) \int_{-\infty}^{x} y_{-}(u) \psi_{1}(u) d u=r_{+}(\varepsilon) r_{-}(\varepsilon) \sum_{k, j=1,2} \tilde{G}_{j, k}(x, \varepsilon),
$$

where

$$
\begin{aligned}
\tilde{G}_{j, k}(x, \varepsilon)= & e^{\varepsilon x} G_{j}^{-}(x ; \varepsilon) \int_{\infty}^{x} e^{-\varepsilon u} G_{k}^{+}(u ; \varepsilon) \psi_{1}(u) d u \\
& -e^{-\varepsilon x} G_{j}^{+}(x ; \varepsilon) \int_{-\infty}^{x} e^{\varepsilon u} G_{k}^{-}(u ; \varepsilon) \psi_{1}(u) d u
\end{aligned}
$$

$G_{1}^{ \pm}(x ; \varepsilon)=\frac{r_{1}^{ \pm}(x) \varepsilon^{m-1} \ln \varepsilon}{1+\varepsilon\langle x\rangle}$, and $G_{2}^{ \pm}(x ; \varepsilon)=r_{0}^{ \pm}(x)+R_{0}^{ \pm}(x ; \varepsilon)$. By Proposition 10 clearly (84) holds with $G_{2}^{ \pm}$instead of $R_{0}^{ \pm}$. We denote for $(j, k)=(0,1)$ or $(1,0)$

$$
\hat{G}_{j, k}(x)=\left(r_{j}^{-}(x) \int_{\infty}^{x} r_{k}^{+}(u) \psi_{1}(u) d u-r_{j}^{+}(x) \int_{-\infty}^{x} r_{k}^{-}(u) \psi_{1}(u) d u\right) .
$$

By direct calculation for $0 \leqslant k \leqslant m-1$,

$$
\begin{array}{r}
\left|\frac{\partial^{k}}{\partial \varepsilon^{k}}\left(\tilde{G}_{2,1}(x, \varepsilon)-\varepsilon^{m-1} \ln \varepsilon \hat{G}_{0,1}(x)\right)\right| \lesssim \sum_{\substack{i+j+l=k \\
i<m-1}}|\varepsilon|^{m-1-i-\delta}\langle x\rangle^{1+j}\left\|\langle x\rangle^{2+l} \psi_{1}(x)\right\|_{1} \\
\\
\lesssim\langle x\rangle^{1+k}\left\|\langle x\rangle^{2+k} \psi_{1}(x)\right\|_{1},
\end{array}
$$


(103)

$$
\begin{array}{r}
\left|\frac{\partial^{m}}{\partial \varepsilon^{m}}\left(\tilde{G}_{2,1}(x, \varepsilon)-\varepsilon^{m-1} \ln \varepsilon \hat{G}_{0,1}(x)\right)\right| \lesssim \sum_{\substack{i+j+l=m \\
i<m}}|\varepsilon|^{m-1-i-\delta}\langle x\rangle^{1+j}\left\|\langle x\rangle^{2+l} \psi_{1}(x)\right\|_{1} \\
\lesssim|\varepsilon|^{-\delta}\langle x\rangle^{1+m}\left\|\langle x\rangle^{2+m} \psi_{1}(x)\right\|_{1},
\end{array}
$$

and it can be similarly shown that for $0 \leqslant k \leqslant m$,

$$
\left|\frac{\partial^{k}}{\partial \varepsilon^{k}}\left(\tilde{G}_{1,2}(x, \varepsilon)-\varepsilon^{m-1} \ln \varepsilon \hat{G}_{1,0}(x)\right)\right| \lesssim\left(1+|\varepsilon|^{m-k-\delta}\right)\langle x\rangle^{2+k}\left\|\langle x\rangle^{1+k} \psi_{1}(x)\right\|_{1} .
$$

Also, for $0 \leqslant k \leqslant m$

$$
\begin{gathered}
\left|\frac{\partial^{k}}{\partial \varepsilon^{k}} \tilde{G}_{1,1}(x, \varepsilon)\right| \lesssim \sum_{i+j+l=k} \frac{|\varepsilon|^{2 m-2-i}(|\ln \varepsilon|+1)^{2}\langle x\rangle^{2+j}}{1+|\varepsilon|\langle x\rangle}\left\|\frac{\langle x\rangle^{2+l} \psi_{1}(x)}{1+|\varepsilon|\langle x\rangle}\right\|_{1} \\
\quad \lesssim\langle x\rangle^{1+k}\left\|\langle x\rangle^{1+k} \psi_{1}(x)\right\|_{1}, \\
\left|\frac{\partial^{k}}{\partial \varepsilon^{k}} \tilde{G}_{2,2}(x, \varepsilon)\right| \lesssim \sum_{i+j+l=k}\left(|\varepsilon|^{m-i-\delta}+1\right)\langle x\rangle^{1+j}\left\|\langle x\rangle^{1+l} \psi_{1}(x)\right\|_{1} .
\end{gathered}
$$

Using (102)-(105) as well as (89) we have

$$
\mathcal{G}\left(\psi_{1}\right)=\frac{r_{+}(\varepsilon) r_{-}(\varepsilon) \sum_{k, j=1,2} \tilde{G}_{j, k}(x, \varepsilon)}{W(\varepsilon)}=r_{3}(x) \varepsilon^{m-1} \ln \varepsilon+\hat{R}(x, \varepsilon),
$$

where $r_{3}(x)=\left(\hat{G}_{0,1}(x)+\hat{G}_{1,0}(x)\right) / W(0)$ satisfies (97) and (99) holds with $\hat{R}$ replacing $R$. (106) and (96) give

$$
R(x, \varepsilon)-\hat{R}(x, \varepsilon)=r_{3}(x) \varepsilon^{m-1} \ln \varepsilon\left(1-\frac{1}{(1+\varepsilon\langle x\rangle)^{m+2}}\right)-\mathcal{G}_{0}\left(\psi_{1}\right) .
$$

Now by direct calculation (99) holds with the right side of (107) replacing $R$, using

$$
\left|\int_{ \pm \infty}^{x}(u-x)^{k} e^{\mp \varepsilon(u-x)} \psi_{1}(u) d u\right| \lesssim\langle x\rangle^{k}\left\|\langle x\rangle^{k} \psi_{1}(x)\right\|_{1}
$$

for the estimates involving $\mathcal{G}_{0}(\psi)$. Thus $(\underline{96)}$ is valid for $|\varepsilon| \leqslant 1$.

For $|\varepsilon| \geqslant 1$, we analyze the numerator of (5) by noting that (recall that $f_{ \pm}=$ $\left.1+s_{ \pm}\right)$

$$
\begin{aligned}
& f_{-}(u ; \varepsilon) \int_{\infty}^{x} e^{-\varepsilon(u-x)} f_{+}(u ; \varepsilon) \psi_{1}(u) d u=\int_{\infty}^{x} e^{-\varepsilon(u-x)} \psi_{1}(u) d u \\
& \quad+s_{-}(x ; \varepsilon) \int_{\infty}^{x} e^{-\varepsilon(u-x)} f_{+}(u ; \varepsilon) \psi_{1}(u) d u+\int_{\infty}^{x} e^{-\varepsilon(u-x)} s_{+}(u ; \varepsilon) \psi_{1}(u) d u
\end{aligned}
$$

and $s_{ \pm}$satisfies (86). The same type of expansion works for the integral from $-\infty$ to $x$. Thus by (5) we have

$$
\mathcal{G}\left(\psi_{1}\right)=\frac{2 \varepsilon \mathcal{G}_{0}\left(\psi_{1}\right)+(1+\varepsilon) R_{3}(x ; \varepsilon)}{W(\varepsilon)}
$$

for some $R_{3}$ satisfying estimates of the type in (99). Note that $W(\varepsilon)$ satisfies (90)). Obviously (99) holds with $r_{3}(x) \frac{\varepsilon^{m-1} \ln \varepsilon}{(1+\varepsilon\langle x\rangle)^{m+2}}$ replacing $R$. Thus (96) is valid for $|\varepsilon| \geqslant 1$ as well. 
Similarly we have

Proposition 12. For $\varepsilon \in \overline{\mathbb{H}} \backslash\{0\}$ we have

$$
\begin{aligned}
\mathcal{G}\left(\varepsilon \psi_{0}\right)= & r_{4}(x) \frac{\varepsilon^{m} \ln \varepsilon}{(1+\varepsilon\langle x\rangle)^{m+3}}-\frac{\psi_{0}(x) \varepsilon}{(\varepsilon+1)^{2}}+\frac{\varepsilon}{\varepsilon+1} \mathcal{G}_{0}\left(\psi_{0}(x)\right) \\
& +\frac{\varepsilon}{\varepsilon+1} \mathcal{G}_{1}\left(\psi_{0}^{\prime}(x)\right)+R_{4}(x ; \varepsilon)
\end{aligned}
$$

where

$$
\begin{gathered}
\left\|\langle x\rangle^{-2} r_{4}(x)\right\|_{\infty} \lesssim\left\|\langle x\rangle^{2} \psi_{0}(x)\right\|_{1}, \\
\left\|\psi_{0}(x)\right\|_{\infty} \lesssim\left\|\psi_{0}^{\prime}(x)\right\|_{1}, \\
\mathcal{G}_{1}(\psi):=\frac{1}{2(1+\varepsilon)}\left(\int_{\infty}^{x} e^{-\varepsilon(u-x)} \psi(u) d u+\int_{-\infty}^{x} e^{\varepsilon(u-x)} \psi(u) d u\right),
\end{gathered}
$$

$$
\begin{array}{r}
\left\|\langle x\rangle^{-k-2} \frac{\partial^{k}}{\partial \varepsilon^{k}} R_{4}(x ; \varepsilon)\right\|_{\infty} \lesssim(1+|\varepsilon|)^{-2}\left(\left\|\langle x\rangle^{k+2} \psi_{0}(x)\right\|_{1}+\left\|\langle x\rangle^{k+2} \psi_{0}^{\prime}(x)\right\|_{1}\right) \\
(0 \leqslant k \leqslant m), \\
\left\|\langle x\rangle^{-m-3} \frac{\partial^{m+1}}{\partial \varepsilon^{m+1}} R_{4}(x ; \varepsilon)\right\|_{\infty} \lesssim\left(|\varepsilon|^{\delta}+|\varepsilon|\right)^{-2}\left(\left\|\langle x\rangle^{m+3} \psi_{0}(x)\right\|_{1}+\left\|\langle x\rangle^{m+3} \psi_{0}^{\prime}(x)\right\|_{1}\right) .
\end{array}
$$

Proof. The proof is essentially the same as that of Proposition [1] In the case $|\varepsilon| \leqslant 1$, we simply use (100)-(105) with $\psi_{1}$ replaced by $\varepsilon \psi_{0}$ for $0 \leqslant k \leqslant m+1$, which gives the counterpart of (106) as

$$
\mathcal{G}\left(\varepsilon \psi_{0}\right)=r_{4}(x) \varepsilon^{m} \ln \varepsilon+\hat{R}_{4}(x, \varepsilon),
$$

where $r_{4}$ satisfies (110) and $\hat{R}_{4}$ satisfies the same estimates as $R_{4}$ (see (111)). The rest follows in the same way as (107). Note that we have the obvious inequality $\left|\psi_{0}(x)\right| \lesssim\left\|\psi_{0}^{\prime}(x)\right\|_{1}$.

For $|\varepsilon| \geqslant 1$ we use integration by parts to get

$$
\begin{gathered}
\varepsilon e^{\varepsilon x} \int_{\infty}^{x} y_{+}(u) \psi_{0}(u) d u=\varepsilon e^{\varepsilon x} \int_{\infty}^{x} e^{-(\varepsilon+1) u} e^{u}\left(1+s_{+}(u ; \varepsilon)\right) \psi_{0}(u) d u \\
=-\frac{\varepsilon}{\varepsilon+1}\left(1+s_{+}(x ; \varepsilon)\right) \psi_{0}(x)+\frac{\varepsilon}{\varepsilon+1} e^{\varepsilon x} \int_{\infty}^{x} e^{-\varepsilon u}\left(\psi_{0}^{\prime}(u)+\psi_{0}(u)\right) d u \\
\quad+\frac{\varepsilon}{\varepsilon+1} e^{\varepsilon x} \int_{\infty}^{x} e^{-\varepsilon u}\left(s_{+}^{\prime}(u ; \varepsilon) \psi_{0}(u)+s_{+}(u ; \varepsilon)\left(\psi_{0}^{\prime}(u)+\psi_{0}(u)\right)\right) d u
\end{gathered}
$$

Similarly

$$
\begin{gathered}
-\varepsilon e^{-\varepsilon x} \int_{-\infty}^{x} y_{-}(u) \psi_{0}(u) d u=-\frac{\varepsilon}{\varepsilon+1}\left(1+s_{-}(x ; \varepsilon)\right) \psi_{0}(x) \\
\quad+\frac{\varepsilon}{\varepsilon+1} e^{-\varepsilon x} \int_{-\infty}^{x} e^{\varepsilon u}\left(\psi_{0}^{\prime}(u)-\psi_{0}(u)\right) d u \\
+\frac{\varepsilon}{\varepsilon+1} e^{-\varepsilon x} \int_{-\infty}^{x} e^{\varepsilon u}\left(s_{-}^{\prime}(u ; \varepsilon) \psi_{0}(u)+s_{-}(u ; \varepsilon)\left(\psi_{0}^{\prime}(u)-\psi_{0}(u)\right)\right) d u
\end{gathered}
$$


Since $s_{ \pm}$satisfy (86), by (15), (113) and (114) we have

$$
\mathcal{G}\left(\varepsilon \psi_{0}\right)=-\frac{2 \varepsilon \psi_{0}(x)}{(\varepsilon+1) W(\varepsilon)}+\frac{2 \varepsilon \mathcal{G}_{0}\left(\psi_{0}(x)\right)+\mathcal{G}_{1}\left(\psi_{0}^{\prime}(x)\right)}{W(\varepsilon)}+\frac{\varepsilon \tilde{R}_{3}(x ; \varepsilon)}{W(\varepsilon)}
$$

for some $\tilde{R}_{3}$ satisfying estimates of the type (111). The rest follows from (90).

\section{TIME DECAY OF $\psi$}

Proof of Theorem 1. We focus on the case $\frac{\sin (t \sqrt{A})}{\sqrt{A}} \psi_{1}$ since the proof for $\cos (t \sqrt{A}) \psi_{0}$ is analogous.

We have by using the decomposition (96) that

$$
\begin{aligned}
& \psi(x, t)=\mathcal{L}^{-1} \hat{\psi}(x, \varepsilon) \\
& =\frac{1}{2 \pi i} \int_{-\infty i}^{\infty i} e^{\varepsilon t}\left(r_{3}(x) \frac{\varepsilon^{m-1} \ln \varepsilon}{(1+\varepsilon\langle x\rangle)^{m+1}}+R(x ; \varepsilon)\right) d \varepsilon+\mathcal{L}^{-1} \mathcal{G}_{0}\left(\psi_{1}(x)\right),
\end{aligned}
$$

where the inverse Laplace transform $\mathcal{L}^{-1}$ can be represented using the Bromwich integral formula for the terms $r_{3}(x) \frac{\varepsilon^{m-1} \ln \varepsilon}{(1+\varepsilon\langle x\rangle)^{m+1}}$ and $R$, and we have

Lemma 13. We have the estimate

$$
\left|\mathcal{L}^{-1} \mathcal{G}_{0}\left(\psi_{1}(x)\right)\right| \lesssim(\langle x\rangle /\langle t\rangle)^{m+1}\left\|\langle x\rangle^{m+1} \psi_{1}(x)\right\|_{1} .
$$

Proof. By direct calculation

$$
\mathcal{G}_{0}\left(\psi_{1}(x)\right)=\mathcal{L}\left(\frac{1}{2} \int_{t+x}^{x} e^{-t+u-x} \psi_{1}(u) d u-\frac{1}{2} \int_{x-t}^{x} e^{-t-u+x} \psi_{1}(u) d u\right) .
$$

The estimate (116) follows from integration by parts. For instance we have

$$
\begin{array}{r}
\int_{t+x}^{x} e^{-t+u-x} \psi_{1}(u) d u=\left.\frac{e^{-t+u-x}}{\left(\sqrt{u^{2}+1}\right)^{m+1}}\right|_{t+x} ^{x} \int_{t+x}^{x}\left(\sqrt{u^{2}+1}\right)^{m+1} \psi_{1}(u) d u \\
-\int_{t+x}^{x}\left(\frac{e^{-t+u-x}}{\left(\sqrt{u^{2}+1}\right)^{m+1}}\right)^{\prime}\left(\int_{0}^{u}\left(\sqrt{v^{2}+1}\right)^{m+1} \psi_{1}(v) d v\right) d u
\end{array}
$$

where the first term on the right side can be estimated using the elementary inequality

$$
\frac{1}{\left(\sqrt{(t+x)^{2}+1}\right)^{m+1}} \lesssim(\langle x\rangle /\langle t\rangle)^{m+1}
$$

and the last term can be estimated by noting that

$$
\begin{aligned}
& \left|\int_{t+x}^{x}\left(\frac{e^{-t+u-x}}{\left(\sqrt{u^{2}+1}\right)^{m+1}}\right)^{\prime} d u\right| \leqslant\left|\int_{t+x}^{x} \frac{e^{-t+u-x}}{\left(\sqrt{u^{2}+1}\right)^{m+1}} d u\right| \leqslant \int_{x}^{x+t / 2} \frac{e^{-t+u-x}}{\left(\sqrt{u^{2}+1}\right)^{m+1}} d u \\
& +\int_{x+t / 2}^{x+t} \frac{e^{-t+u-x}}{\left(\sqrt{u^{2}+1}\right)^{m+1}} d u \lesssim e^{-t / 2}+\sup _{t / 2 \leqslant v \leqslant t} \frac{1}{\left(\sqrt{(x+v)^{2}+1}\right)^{m+1}} \lesssim(\langle x\rangle /\langle t\rangle)^{m+1} .
\end{aligned}
$$


The leading term on the right hand side of (115) can be estimated by contour deformation. Since there is a branch cut along $\mathbb{R}^{-}$, the original integration path from $-i \infty$ to $i \infty$ can be deformed into the path consisting of the vertical line from $-1 / 2-i \infty$ to $-1 / 2$, the horizonal line from $-1 / 2$ to 0 on the lower side of the branch cut, the horizonal line from 0 to $-1 / 2$ on the upper side of the branch cut, and the vertical line from $-1 / 2$ to $-1 / 2+i \infty$. Therefore, by Watson's lemma we have for large $t$

$$
\begin{aligned}
\frac{1}{2 \pi i} \int_{-\infty i}^{\infty i} e^{\varepsilon t} r_{3}(x) & \frac{\varepsilon^{m-1} \ln \varepsilon}{(1+\varepsilon\langle x\rangle)^{m+1}} d \varepsilon \\
= & -\int_{0}^{-1 / 2} e^{\varepsilon t} \frac{r_{3}(x) \varepsilon^{m-1}}{(1+\varepsilon\langle x\rangle)^{m+1}} d \varepsilon+O\left(e^{-t / 2}\right) r_{3}(x) \\
= & (-1)^{m+1}(m-1) ! r_{3}(x) t^{-m}\left(1+O\left(t^{-1}\right)\langle x\rangle\right)+O\left(e^{-t / 2}\right) r_{3}(x),
\end{aligned}
$$

Note that the left side of (119) is obviously bounded by const. $\left|r_{3}(x)\right|$ uniformly for all $t \geqslant 0$ and $r_{3}$ satisfies (97). Finally, by integration by parts and (99) we have for $t>0$

$$
\left|\frac{1}{2 \pi i} \int_{-\infty i}^{\infty i} e^{\varepsilon t} R(x ; \varepsilon) d \varepsilon\right| \lesssim t^{-m}\left|\int_{-\infty i}^{\infty i} e^{\varepsilon t} \frac{\partial^{m} R(x ; \varepsilon)}{\partial \varepsilon^{m}} d \varepsilon\right|
$$

Since $R(x ; \varepsilon)$ and $\frac{\partial^{m} R(x ; \varepsilon)}{\partial \varepsilon^{m}}$ satisfy $(\underline{99)}$, we have for $t \geqslant 0$

$$
\left\|\langle x\rangle^{-m-2} \mathcal{L}^{-1} R(x ; \varepsilon)\right\|_{\infty} \lesssim\langle t\rangle^{-m}\left\|\langle x\rangle^{m+2} \psi_{1}(x)\right\|_{1} .
$$

Furthermore, it follows from the Riemann-Lebesgue lemma that

$$
\lim _{t \rightarrow \infty} t^{m} \mathcal{L}^{-1} R(x ; \varepsilon)=0 .
$$

We define $\hat{r}_{1}(x)=(-1)^{m+1}(m-1) ! r_{3}(x)$ and

$$
R_{1}(x, t)=\langle t\rangle^{m} \mathcal{L}^{-1}\left(\mathcal{G}_{0}\left(\psi_{1}(x)\right)+R(x ; \varepsilon)+r_{3}(x) \frac{\varepsilon^{m-1} \ln \varepsilon}{(1+\varepsilon\langle x\rangle)^{m+1}}\right)-\hat{r}_{1}(x) .
$$

The result for $\frac{\sin (t \sqrt{A})}{\sqrt{A}} \psi_{1}$ in Theorem 1 then follows from (115) using (116), (119), (120), and (121); for $\cos (t \sqrt{A}) \psi_{0}$ the estimates follow from Proposition 12 in a similar way.

\subsection{Genericity of decay rate.}

Remark 6. In view of the definition of $r_{3}$ (below (106) ) it is clear that $r_{3}$ is nonzero for generic initial condition $\psi_{1}$, meaning the time decay $t^{-m}$ is generic.

\section{More General Potentials}

7.1. Sums of inverse powers. Assume $V(x)=$ const. $x^{-\alpha_{1}^{ \pm}}\left(1+\sum_{k=1}^{n} a_{k}^{ \pm} x^{-\beta_{k}^{ \pm}}\right)$ for large $\pm x$ where $\alpha_{1}^{ \pm}>2$ and $\beta_{k}^{ \pm}>0$. 
Without loss of generality we study large $x$. Now (9) has the form

$$
\begin{aligned}
F(\tau)= & \text { const. }(\varepsilon \tau)^{\alpha_{1}^{+}-1}\left(1+\sum_{k=1}^{n} b_{k}(\varepsilon \tau)^{\beta_{k}^{+}}\right) \\
& + \text {const. } \varepsilon^{\alpha_{1}^{+}-2} \int_{0}^{\tau}(\tau-u)^{\alpha_{1}^{+}-1}\left(1+\sum_{k=1}^{n} b_{k}(\varepsilon(\tau-u))^{\beta_{k}^{+}}\right) \frac{F(u)}{u(u+2)} d u .
\end{aligned}
$$

It can be shown that for large $\tau$

$$
\begin{aligned}
& \frac{F(\tau)}{\varepsilon^{2} \tau(\tau+2)}=\text { const. }(\varepsilon \tau)^{\alpha_{1}^{+}-3}\left(1+\sum_{k=1}^{n} b_{k}(\varepsilon \tau)^{\beta_{k}^{+}}\right) \\
& \times\left(1+\sum_{k=0}^{n} \sum_{l=1}^{\infty} \sum_{m=0}^{l} c_{k l m}(\varepsilon \tau)^{l\left(\alpha_{1}^{+}-2+\beta_{k}^{+}\right)}(\ln \tau)^{m}\right),
\end{aligned}
$$

where $\beta_{0}=0$ and the ln terms are only present for $\alpha_{1}^{+} \in \mathbb{N}$.

The counterpart of (35) is now the expansion

$$
s(x ; \varepsilon)=\varepsilon^{\alpha_{1}^{+}-2} \sum_{k=0}^{n}\left(\tilde{c}_{k}(x)+\varepsilon c_{k}(x)\right) \varepsilon^{\beta_{k}^{+}}+\varepsilon^{\alpha_{1}^{+}-2} \ln \varepsilon \sum_{k=0}^{n}\left(\tilde{C}_{k}(x)+\varepsilon C_{k}(x)\right) \varepsilon^{\beta_{k}^{+}}+\ldots,
$$

where terms with higher orders of $\ln$ are omitted and the $\ln$ terms are only present for $\alpha_{1}^{+} \in \mathbb{N}$.

This implies the counterpart of the expansion (80),

$$
y_{+}(x ; \varepsilon)=r(\varepsilon) e^{-\varepsilon x}\left(\hat{D}_{1}(x) \varepsilon^{\alpha_{1}^{+}-1}+\hat{D}_{2}(x) \varepsilon^{\alpha_{1}^{+}-1} \ln \varepsilon+R(x ; \varepsilon)\right),
$$

where $\frac{\partial^{k} R(x ; \varepsilon)}{\partial \varepsilon^{k}}$ are bounded by $\varepsilon^{\delta-1}$ for $0 \leqslant k \leqslant\left\lceil\alpha_{1}^{+}\right\rceil$and some $\delta>0$, and $D_{2}=0$ except for $\alpha_{1} \in \mathbb{N}$.

Arguments similar to those showing Proposition 11 lead to the same type of expansion for $\hat{\psi}$, which then implies that

$$
\begin{gathered}
\frac{\sin (t \sqrt{A})}{\sqrt{A}} \psi_{1} \sim \hat{r}_{1}(x) t^{-\alpha}, \\
\cos (t \sqrt{A}) \psi_{0} \sim \hat{r}_{0}(x) t^{-\alpha-1} .
\end{gathered}
$$

The detailed estimates for the higher order remainder as in Theorem 1 can be obtained in similar ways.

7.2. Inverse power with higher order correction. Here we discuss the general $m$ analog of potentials of the type in [12. Assume $V(x)=V_{0}(x)+V_{1}(x)$ and $V_{0}(x)=c_{v} / x^{m}$ for $|x| \geqslant x_{+}, V_{1}$ is piecewise continuous, $\left|V_{1}^{(k)}(x)\right| \lesssim\langle x\rangle^{-m-k-\delta_{1}}$ where $0 \leqslant k \leqslant m+2$ and $\delta_{1}>3$. One can show that for $x \geqslant x_{+}$and $|\varepsilon| \leqslant 1 / x, \tilde{y}_{1}$ has the expansion

$$
\tilde{y}_{1}(x ; \varepsilon)=r(\varepsilon)\left(\tilde{B}_{0}(x)+\tilde{B}_{1}(x) \varepsilon^{m-1} \ln \varepsilon+f_{d}(x, \varepsilon)\right),
$$

where $\tilde{B}_{k}$ solves $f^{\prime \prime}(x)=V(x) f(x)$ with $\tilde{B}_{0} \sim \Phi_{1}=: B_{0}, \tilde{B}_{1} \sim B_{1}$ for large $x$ and $f_{d}(x, \varepsilon)$ satisfies (57). 
Indeed, by (55) and standard ODE analysis one can find $\tilde{B}_{k}(x)$ with $\tilde{B}_{k}^{\prime \prime}(x)=$ $V(x) \tilde{B}_{k}(x), \quad\left|\tilde{B}_{k}(x)-B_{k}(x)\right| \lesssim\langle x\rangle^{2-m-\delta_{1}}\left\langle B_{k}(x)\right\rangle$ and $\left|\tilde{B}_{k}^{\prime}(x)-B_{k}^{\prime}(x)\right| \lesssim$ $\langle x\rangle^{1-m-\delta_{1}}\left\langle B_{k}^{\prime}(x)\right\rangle$.

By (55) we have

$$
f_{+}(x ; \varepsilon)=r(\varepsilon)\left(\hat{\Phi}_{1}(x)+B_{1}(x) \varepsilon^{m-1} \ln \varepsilon+f_{c}(x, \varepsilon)\right)=: r(\varepsilon)\left(\phi(x ; \varepsilon ; B)+f_{c}(x, \varepsilon)\right),
$$

where $f_{c}$ satisfies (57) and

$$
f_{c}^{\prime \prime}(x, \varepsilon)-2 \varepsilon f_{c}^{\prime}(x, \varepsilon)-V_{0}(x) f_{c}(x, \varepsilon)=2 \varepsilon \phi\left(x ; \varepsilon ; B^{\prime}\right) .
$$

Similarly we write $\tilde{f}_{1}(x ; \varepsilon)=r(\varepsilon)\left(\phi(x ; \varepsilon ; \tilde{B})+f_{c}(x, \varepsilon)+g_{1}(x, \varepsilon)\right)$, which implies that $\tilde{g}_{1}(x, \varepsilon)=e^{-\varepsilon x} g_{1}(x, \varepsilon)$ satisfies the equation

$$
\tilde{g}_{1}^{\prime \prime}(x, \varepsilon)-\varepsilon^{2} \tilde{g}_{1}(x, \varepsilon)-V_{0}(x) \tilde{g}_{1}(x, \varepsilon)=e^{-\varepsilon x} \phi_{1}(x, \varepsilon)+V_{1}(x) \tilde{g}_{1}(x, \varepsilon),
$$

where $\phi_{1}(x, \varepsilon)=2 \varepsilon \phi\left(x ; \varepsilon ; \tilde{B}^{\prime}-B^{\prime}\right)+\varepsilon V_{1}(x) \hat{f}_{c}(x, \varepsilon)$ and $\hat{f}_{c}=f_{c} / \varepsilon$. Equivalently we have the integral equation

$$
g_{1}(x)=\mathcal{G}\left(e^{-\varepsilon x} \phi_{1}(x ; \varepsilon)\right)+\mathcal{G}\left(V_{1}(x) g_{1}(x)\right) .
$$

Using Proposition 10 we see that $\left|\phi_{1}(x ; \varepsilon)\right| \lesssim|\varepsilon|\langle x\rangle^{1-m-\delta_{1}}$ for all $x \geqslant x_{+}$and thus (126) is contractive under the norm $\left\|\langle x\rangle^{m-2+\delta_{1}} g_{1}(x)\right\|_{\infty}$, and by taking derivatives of $(126)$ we have

$$
\begin{gathered}
\left|\frac{\partial^{k} g_{1}(x ; \varepsilon)}{\partial \varepsilon^{k}}\right| \lesssim x^{2-m-\delta_{1}+k}(0 \leqslant k \leqslant m-1) ; \\
\left|\frac{\partial^{k} g_{1}(x ; \varepsilon)}{\partial \varepsilon^{k}}\right| \lesssim|\varepsilon|^{m-k-\delta} x^{2-\delta_{1}} \quad(m \leqslant k \leqslant m+1)
\end{gathered}
$$

since

$$
\left|e^{\varepsilon x} \int_{\infty}^{x} e^{-2 \varepsilon t} t^{k} \phi_{1}(t ; \varepsilon) d t\right| \lesssim 1
$$

if $k-m-\delta_{1}<-2$. Thus if $\delta_{1}>3$, then the counterpart of Proposition 7 holds. The rest of the proof is similar.

\section{Appendix}

For completeness, in this section we provide a short self-contained proof justifying the use of the Laplace transform.

Proposition 14. Assume the initial conditions $f(x)=u(x, 0)$ and $g(x)=u_{t}(x, 0)$ are in $L^{1}(\mathbb{R})$ and $V \in L^{\infty}(\mathbb{R})$. Then, if $\nu>\sqrt{2}\|V\|_{\infty}^{\frac{1}{2}}$ we have $\sup _{t>0} e^{-\nu t}\|u(t, \cdot)\|_{1}$ $<\infty$, and thus $u(t, x)$ is Laplace transformable in $t$ for $\operatorname{Re} t>\nu$.

Proof. We use the Duhamel principle to write (10) in the form

$$
\begin{aligned}
u=\mathcal{A} u ; \mathcal{A} u:=\frac{f(x-t)+f(x+t)}{2} \\
\left.\quad+\frac{1}{2} \int_{-\infty}^{\infty} \chi_{t}(y-x) g(y) d y+\frac{1}{2} \int_{0}^{t} \int_{-\infty}^{\infty} u(y, s) V(y) \chi_{t-s}(y-x)\right) d y d s
\end{aligned}
$$


where $\chi_{a}$ is the characteristic function of the interval $[-a, a]$. Consider the Banach space

$$
\mathcal{B}=\left\{u \in C(\mathbb{R}) \mid\|u\|_{\nu}:=\sup _{t \in \mathbb{R}^{+}} e^{-\nu t}\|u(t, \cdot)\|_{1}<\infty\right\} \quad\left(\nu>\sqrt{2}\|V\|_{\infty}^{\frac{1}{2}}\right) .
$$

Applying Fubini to integrate first in $x$, we see that $\left\|\int_{-\infty}^{\infty} \chi_{t}(y-x) g(y) d y\right\|_{1} \leqslant 2 t\|g\|_{1}$ and (since by definition $\|u(\cdot, s)\|_{1} \leqslant\|u\|_{\nu} e^{\nu s}$ )

$$
\begin{aligned}
& \sup _{t>0} e^{-\nu t}\left\|\int_{0}^{t} \int_{-\infty}^{\infty} u(y, s) V(y) \chi_{t-s}(y-x) d y d s\right\|_{1} \\
& \quad \leqslant y\|V\|_{\infty}\|u\|_{\nu} \sup _{t>0} e^{-\nu t} \int_{0}^{t} 2(t-s) e^{\nu s} d s \leq 2\|V\|_{\infty} \nu^{-2}\|u\|_{\nu} .
\end{aligned}
$$

Using (129) we see that $\mathcal{A}: \mathcal{B} \rightarrow \mathcal{B}$ is contractive. Also, assuming $f, g$ and $V$ are smooth, the solution is seen to be smooth too: since $u \in L^{1}$, Duhamel's formula shows that it is continuous; then, as usual, using continuity we derive differentiability, and, inductively, we see that $u$ is smooth.

Proof of Lemma 6. This is by straightforward calculation. For $n \geqslant 0$ we get

$$
\begin{aligned}
\int_{3}^{\infty} e^{-\varepsilon \tau x} \tau^{n}(\ln \tau)^{l} d \tau & =\left(\int_{0}^{\infty}-\int_{0}^{3}\right) e^{-\varepsilon \tau x} \tau^{n}(\ln \tau)^{l} d \tau \\
& =\frac{1}{(\varepsilon x)^{n+1}} \int_{0}^{\infty} e^{-u} u^{n}(\ln u-\ln (\varepsilon x))^{l} d u-\int_{0}^{3} e^{-\varepsilon \tau x} \tau^{n}(\ln \tau)^{l} d \tau \\
& =\frac{1}{(\varepsilon x)^{n+1}} \sum_{q=0}^{l} c_{q}^{(n ; l)}(\ln (\varepsilon x))^{q}-\int_{0}^{3} e^{-\varepsilon \tau x} \tau^{n}(\ln \tau)^{l} d \tau
\end{aligned}
$$

where the last term is $R_{a, n}$ and (46) is immediate, and

$$
c_{q}^{(n ; l)}=\left(\int_{0}^{\infty} e^{-u} u^{n}(\ln u)^{l-q} d u\right) \frac{(-1)^{q} l !}{(l-q) ! q !} .
$$

In particular, $c_{1}^{(0 ; 1)}=-1$.

Now (47) for $n=-1$ follows from integration by parts:

$$
\int_{3}^{\infty} e^{-\varepsilon \tau x} \tau^{-1}(\ln \tau)^{l} d \tau=-\frac{1}{l+1} e^{-3 \varepsilon x}(\ln 3)^{l+1}+\frac{\varepsilon x}{l+1} \int_{3}^{\infty} e^{-\varepsilon \tau x}(\ln \tau)^{l+1} d \tau,
$$

where the first term satisfies (46) and the last integral in (131) was evaluated in (130).

For $n<-1$ we have by integration by parts:

$$
\begin{aligned}
& \int_{3}^{\infty} e^{-\varepsilon \tau x} \tau^{n}(\ln \tau)^{l} d \tau=-\frac{1}{n+1} e^{-3 \varepsilon x} 3^{n+1}(\ln 3)^{l} \\
& \quad-\frac{l}{n+1} \int_{3}^{\infty} e^{-\varepsilon \tau x} \tau^{n}(\ln \tau)^{l-1} d \tau+\frac{\varepsilon x}{n+1} \int_{3}^{\infty} e^{-\varepsilon \tau x} \tau^{n+1}(\ln \tau)^{l} d \tau
\end{aligned}
$$

and (47) follows by induction on $l$ and $n$ using (132) and integration by parts. 


\section{ACKNOWLEDGMENTS}

This work was supported in part by the National Science Foundation DMS01108794 (OC).

\section{REFERENCES}

[1] Lars Andersson, Pieter Blue, and Jean-Philippe Nicolas, A decay estimate for a wave equation with trapping and a complex potential, Int. Math. Res. Not. IMRN 3 (2013), 548-561. MR 3021792

[2] Michael Beals, Optimal $L^{\infty}$ decay for solutions to the wave equation with a potential, Comm. Partial Differential Equations 19 (1994), no. 7-8, 1319-1369, DOI 10.1080/03605309408821056. MR:1284812 (95h:35125)

[3] P. Bizon, T. Chmaj, and A. Rostworowski, Anomalously small wave tails in higher dimensions. Physical Review D (Particles, Fields, Gravitation, and Cosmology) 76 (2007), no. 12, 124035.

[4] E. S. C. Ching, P. T. Leung, W. M. Suen, and K. Young, Wave propagation in gravitational systems: Late time behavior, Physical Review D (Particles, Fields, Gravitation, and Cosmology) 52 (1995), no. 4, 2118-2132.

[5] O. Costin and M. Huang, Gamow vectors and Borel summability in a class of quantum systems, J. Stat. Phys. 144 (2011), no. 4, 846-871, DOI 10.1007/s10955-011-0276-x. MR2826622 (2012h:81073)

[6] O. Costin, J. L. Lebowitz, and S. Tanveer, Ionization of Coulomb systems in $\mathbb{R}^{3}$ by time periodic forcings of arbitrary size, Comm. Math. Phys. 296 (2010), no. 3, 681-738, DOI 10.1007/s00220-010-1023-x. MR2628820 (2011h:81080)

[7] Roland Donninger and Wilhelm Schlag, Decay estimates for the one-dimensional wave equation with an inverse power potential, Int. Math. Res. Not. IMRN 22 (2010), 4276-4300, DOI 10.1093/imrn/rnq038. MR2737771 (2011m:35203)

[8] Piero D'ancona and Vittoria Pierfelice, On the wave equation with a large rough potential, J. Funct. Anal. 227 (2005), no. 1, 30-77, DOI 10.1016/j.jfa.2005.05.013. MR2165087 (2006k:35170)

[9] Piero D'Ancona and Luca Fanelli, $L^{p}$-boundedness of the wave operator for the one dimensional Schrödinger operator, Comm. Math. Phys. 268 (2006), no. 2, 415-438, DOI 10.1007/s00220-006-0098-x. MR2259201 (2007j:35177)

[10] Roland Donninger, Wilhelm Schlag, and Avy Soffer, A proof of Price's law on Schwarzschild black hole manifolds for all angular momenta, Adv. Math. 226 (2011), no. 1, 484-540, DOI 10.1016/j.aim.2010.06.026. MR.2735767 (2012d:58043)

[11] Michael Goldberg, Transport in the one-dimensional Schrödinger equation, Proc. Amer. Math. Soc. 135 (2007), no. 10, 3171-3179, DOI 10.1090/S0002-9939-07-08897-1. MR2322747 (2008k:35391)

[12] W. Schlag, Dispersive estimates for Schrödinger operators: a survey, Mathematical aspects of nonlinear dispersive equations, Ann. of Math. Stud., vol. 163, Princeton Univ. Press, Princeton, NJ, 2007, pp. 255-285. MR2333215 (2009k:35043)

[13] Robert S. Strichartz, A priori estimates for the wave equation and some applications, J. Functional Analysis 5 (1970), 218-235. MR0257581 (41 \#2231)

[14] Robert S. Strichartz, Convolutions with kernels having singularities on a sphere, Trans. Amer. Math. Soc. 148 (1970), 461-471. MR0256219 (41 \#876)

[15] W Wasow, Asymptotic expansions for ordinary differential equations, Interscience Publishers, 1968.

Department of Mathematics, The Ohio State University, Columbus, Ohio 43210

Department of Mathematics, City University of Hong Kong, Hong Kong 